\title{
Validation of energy deposition simulations for proton and heavy ion losses in the CERN Large Hadron Collider
}

A. Lechner, ${ }^{*}$ B. Auchmann, ${ }^{\dagger}$ T. Baer, ${ }^{\ddagger}$ C. Bahamonde Castro, R. Bruce, F. Cerutti, L. S. Esposito, A. Ferrari, J. M. Jowett, A. Mereghetti, F. Pietropaolo, S. Redaelli, B. Salvachua, M. Sapinski, ${ }^{\S}$ M. Schaumann, N. V. Shetty, and V. Vlachoudis

European Organization for Nuclear Research (CERN), Esplanade des Particules 1, 1211 Geneva, Switzerland

E. Skordis

University of Liverpool, Liverpool L69 3BX, United Kingdom and European Organization for Nuclear Research (CERN), Esplanade des Particules 1, 1211 Geneva, Switzerland

(Received 18 March 2019; published 11 July 2019)

\begin{abstract}
Monte Carlo shower simulations are essential for understanding and predicting the consequences of beam losses in high-energy proton and ion colliders. Shower simulations are routinely used at CERN for estimating the beam-induced energy deposition, radiation damage, and radioactivity in the Large Hadron Collider (LHC). Comparing these shower simulations against beam loss measurements is an important prerequisite for assessing the predictive ability of model calculations. This paper validates FLUKA simulation predictions of beam loss monitor (BLM) signals against BLM measurements from proton fills at 3.5 and $4 \mathrm{TeV}$ and ${ }^{208} \mathrm{~Pb}^{82+}$ ion fills at $1.38 \mathrm{ATeV}$. The paper addresses typical loss scenarios and loss mechanisms encountered in LHC operation, including proton collisions with dust particles liberated into the beams, halo impact on collimators in the betatron cleaning insertion, proton-proton collisions in the interaction points, and dispersive losses due to bound-free pair production in heavy ion collisions. Model predictions and measured signals generally match within a few tens of percent, although systematic differences were found to be as high as a factor of 3 for some regions and source terms.
\end{abstract}

DOI: 10.1103/PhysRevAccelBeams.22.071003

\section{INTRODUCTION}

During its first physics run (run I, 2009-2013), the Large Hadron Collider (LHC) [1] at CERN successfully stored and collided 3.5 and $4 \mathrm{TeV}$ proton beams as well as ${ }^{208} \mathrm{~Pb}^{82+}$ beams with the same magnetic rigidity $[2,3]$. After a shutdown of about $2 \mathrm{yr}$ for accelerator maintenance, consolidation, and upgrades (2013-2014), run II operation continued at $6.5 \mathrm{TeV}$ for protons and at $2.51 \mathrm{~A} \mathrm{TeV}$ for ${ }^{208} \mathrm{~Pb}^{82+}$ ions. Since then, the LHC achieved the highestever center-of-mass collision energy of more than $1 \mathrm{PeV}$

\footnotetext{
*Anton.Lechner@cern.ch

On leave at PSI, Paul Scherrer Institut, 5232 Villigen PSI, Switzerland.

${ }^{\ddagger}$ Present address: Mirion Technologies, Ruhrstrasse 49, 22761 Hamburg, Germany.

${ }^{\S}$ Present address: GSI Helmholtzzentrum für Schwerionenforschung, Planckstrasse 1, 64291 Darmstadt, Germany.

Published by the American Physical Society under the terms of the Creative Commons Attribution 4.0 International license. Further distribution of this work must maintain attribution to the author(s) and the published article's title, journal citation, and DOI.
}

with ${ }^{208} \mathrm{~Pb}^{82+}$ ions $[3,4]$ and a world record luminosity of $2.1 \times 10^{34} \mathrm{~cm}^{-2} \mathrm{~s}^{-1}$ with protons [5]. The latter value is more than twice the LHC design luminosity.

An intrinsic feature of collider operation is the continuous and unavoidable loss of particles from the beams. The mechanisms driving such losses can be of different nature, from the burnoff of particles in the interaction points (IPs) to collisions with residual gas molecules in the vacuum chambers. In addition, different phenomena such as longrange beam-beam, electron cloud, and space charge effects cause particles to steadily populate the beam tails. Once these particles reach a certain amplitude, they are intercepted in the LHC collimation insertions, where tightly set collimators pose a controlled global aperture bottleneck $[6,7]$. Losses at collimators can also be provoked by orbit variations or optics changes during the cycle. While all these mechanisms give rise to a continuous reduction of the stored intensity, particle losses can also be of an irregular nature. A phenomenon frequently observed in the LHC is collisions with microscopic dust particles falling into the stored beams [8-15]. Dust particle events occur all around the rings and usually last for a few turns. Disturbances arose 
also from other kinds of beam losses developing at specific locations. At the beginning of run II, frequent loss events were detected in one of the arc dipoles. Beam-based aperture scans indicated that the losses were due to a macroscopic obstacle lying at the bottom of the vacuum chamber [16]. While the origin of the obstacle is still unknown, the events could be successfully suppressed by locally steering the stored beam around the obstacle by means of a magnetic bump. In 2017, recurring loss events in another arc cell posed a major performance limitation for the LHC run [17-19]. The loss events showed some similarities to regular dust events but developed persistent loss tails which gave rise to beam instabilities. These events were presumably caused by nitrogen, oxygen, or water macroparticles, residue from accidental air inflow in the 2016-2017 shutdown, which were subject to a phase transition when being heated up by the beam. Beam losses can also be of an accidental nature because of the malfunctioning of equipment. In the LHC, accidental losses were observed multiple times during the injection phase due to failures of the injection kicker magnets [20]. In such cases, the mis-steered beam is intercepted by protection absorbers which prevent damage to the machine. Accidental beam losses can also happen during the extraction phase but were not yet observed with high-intensity beams.

High-energy protons or $\mathrm{Pb}$ ions lost in the LHC give rise to particle showers in vacuum chambers, magnets, collimators, and other equipment. Interactions of $\mathrm{Pb}$ ions differ qualitatively from those of protons, as they result in the fragmentation of the projectile due to hadronic interactions and electromagnetic dissociation. Eventually, the fragments initiate secondary showers similar to high-energy protons. While multiparticle production in inelastic collisions leads to the formation of hadronic cascades, nuclear interactions also give rise to electromagnetic showers through the production of $\pi^{0}$ mesons which decay into energetic $\gamma$ pairs. Most of the energy released in high-energy hadronic interactions is eventually dissipated by the electromagnetic shower component. The ionizing energy loss of charged particles results in the heating of materials, whereas the nonionizing energy loss can lead to the displacement of atoms from the lattice.

Beam losses and the resulting showers adversely affect collider operation, experiments, equipment, and personnel in several ways. For example, they can lead to magnet quenches, i.e., the sudden loss of superconductivity [21]; they contribute to the heat load to the cryogenic system $[22,23]$; they cause long-term radiation damage and aging of equipment components [22-25]; they lead to the production of radioactive isotopes and are therefore a concern for radiation protection [26]; they give rise to background in experiments [27]; and they can induce single-event effects in equipment electronics [28]. In the worst case, if the beam is lost in an uncontrolled way, it can induce destructive damage because of the thermal shock or because of phase transitions if the temperatures are high enough. In order to assess the consequences of beam losses and to develop mitigation strategies, one requires a good understanding of the particle shower development in the collider. The stochastic nature of particle interactions and secondary particle production can naturally be described with Monte Carlo-based simulation techniques. By employing microscopic interaction and transport models, general-purpose Monte Carlo codes like FLUKA [29,30] can describe the development of particle showers in complex simulation geometries. FLUKA is regularly used at CERN for studying particle-matter interactions of high-energy protons and ions in the LHC [19-21,23,25,26,31-45].

Although the physics models in FLUKA have been well benchmarked at the microscopic level (see, e.g., Refs. [30,46,47]), a validation against measured macroscopic observables is essential for estimating the predictive ability of model calculations for complex radiation environments as in the LHC. In this paper, we derive FLUKA predictions of beam loss monitor (BLM) signals and compare them against BLM measurements from LHC run I. The LHC is equipped with almost 4000 BLMs which detect beam losses and can trigger the extraction of the beams in case the signals exceed predefined abort thresholds $[48,49]$. BLMs are installed on LHC magnets, collimators, and other sensitive equipment. The standard loss monitors used at the LHC are ionization chambers filled with $\mathrm{N}_{2}$ gas at slightly higher than atmospheric pressure (1.1 bar) [48,49]. The BLMs have a sensitive volume of about $1.5 \times 10^{-3} \mathrm{~m}^{3}$, which allows for a relatively large solid angle coverage for the detection of secondary showers. Although the BLMs record the peripheral tail of particle showers since they are installed on the outside of accelerator elements (e.g., outside of magnet cryostats), they provide a suitable measurement for benchmarking energy deposition simulations in the collider environment.

First simulation benchmarks against BLM measurements have been carried out before the LHC startup [50-54]. These studies investigated the BLM response to proton, $\gamma$, and neutron irradiation as well as to mixed radiation fields. Since then, a wealth of BLM data have been recorded during LHC operation, permitting a validation of models for real loss cases. In this paper, we address a variety of loss mechanisms regularly encountered in operation. These include proton collisions with dust particles; halo impact on collimators in the betatron cleaning insertion; proton-proton collisions in the interaction points; and bound-free pair production in heavy ion collisions. In most cases, the loss distribution can be well described analytically, while in other cases, like collimation losses, numerical tracking simulations in the accelerator lattice are needed to determine the multiturn loss distribution. We consider only measurements where the relevant loss mechanism yields the dominant contribution to BLM signals. The measurements originate both from regular 
collider operation and from beam loss experiments probing the quench level of superconducting magnets $[21,55,56]$. FLUKA has been used in the analysis of these experiments to determine the energy deposition in magnet coils [21]. At the same time, the experiments enabled a validation of simulation predictions against BLM measurements for wellcontrolled loss conditions. Here we use data from quench tests carried out with 3.5 and $4 \mathrm{TeV}$ proton beams. The other measurements derive from regular proton $(4 \mathrm{TeV})$ and ${ }^{208} \mathrm{~Pb}^{82+}(1.38 \mathrm{~A} \mathrm{TeV})$ fills for luminosity production.

Selected results of the validation studies discussed in this paper, as well as complementary studies at different beam energies and for different types of losses, have been reported previously in Refs. [21,36,40,41,43,57,58]. The present paper summarizes in a more rigorous way the simulation models and methods (Sec. II) and presents a systematic validation across the different source terms (Secs. III and IV). In particular, the paper provides a more profound discussion about the achievable accuracy and about the discrepancies observed in some cases. In addition, the measurement data were reevaluated where possible, for example, by averaging data over more extended time periods, which reduced the measurement uncertainty. A summary and concluding remarks are given in Sec. V.

\section{SIMULATION MODELS AND METHODS}

Simulating hadron and ion-induced showers in highenergy colliders requires an accurate modeling of particle interactions over a wide energy range. FLUKA is a versatile Monte Carlo code which embeds event generators for describing hadron-nucleon, hadron-nucleus, and nucleusnucleus interactions up to cosmic ray energies [29,30, 47,59]. The hadron-nucleus event generator in FLUKA is called PEANUT and employs the Glauber-Gribove cascade formalism with a formation zone to describe high-energy collisions; particle production is simulated with the dual parton model at high energies and by resonance decay at energies below a few GeV. PEANUT includes as well a preequilibrium model, which is complemented by fragmentation, evaporation, Fermi breakup, fission, and $\gamma$ deexcitation models. FLUKA has also been interfaced to DPMJET-III [59], which is an event generator based on the dual parton model able to treat hadron-hadron collisions up to cosmic ray energies. DPMJET is also used for simulating hadronic nucleus-nucleus interactions above $5 \mathrm{GeV} / \mathrm{u}$, while the Relativistic Quantum Molecular Dynamics (RQMD) and Boltzmann Master Equation (BME) models are used for ion interactions at lower energies. The evaporation and deexcitation stages of fragments are simulated with the same models as above. The code simulates a full coupling between the resulting hadronic and electromagnetic cascades, which determine the energy deposition in collider components.

Shower studies for colliders require both a detailed modeling of the accelerator geometry and a description of the phase space distribution of beam particles lost in the machine. This section describes general features of geometry models and source terms for the different loss scenarios studied in this paper. The second part of the section is dedicated to the simulation of BLM signals and related systematic uncertainties.

\section{A. Accelerator model and source terms}

The shower simulations described in this paper are based on realistic three-dimensional geometry models of magnets, collimators, and other bulk accelerator equipment. The different elements are connected by vacuum chambers which provide a continuous description of the accelerator aperture. The aperture model reproduces the nominal dimensions of drift chambers, while short aperture discontinuities like beam position monitors are generally neglected. The beam lines are assembled by means of auxiliary tools $[60,61]$ and are embedded in a three-dimensional model of the LHC tunnel. For each study presented in this paper, a different geometry model is used. The shortest model includes only a few magnets, whereas the largest one describes several hundred meters of beam line.

The models of superconducting magnets incorporate geometrical features like beam screens, cold bores, coils, collars, yokes, thermal shields, and cryostats. Figure 1 shows the rendered model of a LHC arc dipole. To provide sufficient clearance for the circulating beams, the arc dipoles, cold bores, and beam screens have a horizontal curvature, with a bending radius of about $2800 \mathrm{~m}$, which results in a sagitta of about $9.1 \mathrm{~mm}$ over the magnet length. The simulation model approximates this curvature by means of straight-line segments of about $1 \mathrm{~m}$ length, since

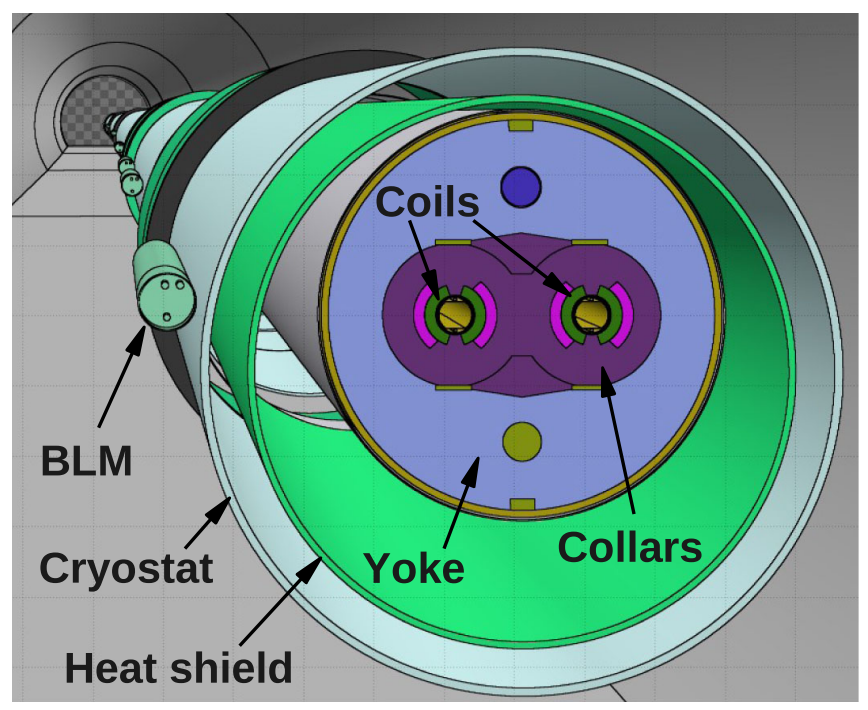

FIG. 1. Geometry model of a main arc dipole embedded in the LHC tunnel, with a BLM mounted on the outside of the magnet cryostat. A more detailed picture of the BLM model is shown in Fig. 2. 
the FLUKA geometry package does not support toroidal segments. Particles can have very grazing angles when impacting on the beam screen, and, hence, the dipole aperture model proves to be critical for accurately describing the loss location in magnets. Compared to a perfect toroidal shape, the straight segment approximation can lead to a local distortion of the particle loss distribution on the beam screen. Depending on the impact angle, the loss location of particles might be displaced by up to a few tens of centimeters in the longitudinal direction. Such a displacement is considered justified, since more important differences between simulated and actual loss locations can arise from aperture imperfections, for example, due to a beam screen or vacuum chamber misalignment. Using 1-mlong segments is also a good compromise between simulation accuracy and computational time, since the latter increases for more complex geometry models.

The coils of most LHC magnets are wound from NbTi Rutherford-type cables which are embedded in superfluid helium. The cables are made of multiple strands, each accommodating several thousands of NbTi filaments. The filaments are embedded in a copper matrix and have a diameter of a few micrometers. It is not feasible to implement such detailed features in the magnet geometry models. The volume occupied by the coils is, therefore, homogeneously filled by a mixture of superconductor, copper stabilizer, insulators, and liquid helium. The material density of such a mixture depends on the magnet type and typically ranges from 6 to $7.2 \mathrm{~g} / \mathrm{cm}^{3}$. For the purpose of our studies, such material mixtures allow for a reasonably accurate description of the particle shower development in magnets, in particular, since the radiation length $X_{0}$ (for example, $\sim 1.9 \mathrm{~cm}$ for NbTi) is much larger than the substructure of strands.

Particle loss locations and particle showers in magnets are strongly affected by the magnetic fields. In all studies, magnetic fields are set according to the beam optics employed during the time of the measurements. The fields of main dipoles and quadrupoles in the arcs and insertion regions are modeled using realistic two-dimensional field maps calculated with ROXIE [62], which extend over the entire magnet cross section. Inside the vacuum chambers, the magnetic field is calculated analytically. The field implementation follows a hard-edge model; i.e., the field maps are applied along the nominal magnetic length of magnets, while fringe fields are neglected. Fields of corrector magnets like orbit correctors or spool pieces are generally set to zero, except for cases where correctors are used to generate local bumps, in particular, the crossing and separation bumps in the experimental insertions. The magnetic settings reproduce the closed beam orbit predicted by the MAD-X optics code [63-65] within a few micrometers.

Depending on the loss scenario, the source term of the shower simulations is either the coordinate distribution of beam particles impacting on the aperture or the coordinate distribution of inelastic proton-proton or proton-nucleus collisions. In the first case, the positions and angles of particles impacting on the aperture are determined by means of other codes like SIXTRACK [66-68]. This method is applied in cases where particles have to be transported to a distant location or where multiturn effects have to be taken into account. In the case of collimation losses, the multiturn tracking simulations also include scattering of beam particles in collimators. The second method is used for scenarios where losses are almost pointlike, e.g., for proton-proton collisions in the interaction points, for losses induced by the wire scanner, or for proton collisions with dust particles.

\section{B. Simulation of BLM signals and related uncertainties}

The cylindrical chambers of standard LHC BLMs are approximately $48 \mathrm{~cm}$ long and have an inner diameter of $8.5 \mathrm{~cm}$. They accommodate 61 circular aluminum electrodes with a radius of $3.75 \mathrm{~cm}$ and a thickness of $0.05 \mathrm{~cm}$, which are alternately connected by means of stainless steel rods (see Fig. 2). Stainless steel spacers keep neighboring electrodes at a distance of $0.575 \mathrm{~cm}$, which yields a nominal active volume of about $1.5 \times 10^{-3} \mathrm{~m}^{3}$. On both ends, the electrodes are enclosed by thick alumina plates, which support the rods and insulate them from other chamber components. A voltage potential of $\sim 1.5 \mathrm{kV}$ is applied to every second electrode, giving rise to a quasiperiodic electric field along the chamber, except in the vicinity of the first and last electrodes. The response time of the monitors is governed by the collection time for ions, which is comparable to the duration of one LHC turn $(\sim 89 \mu \mathrm{s})[48,49]$.
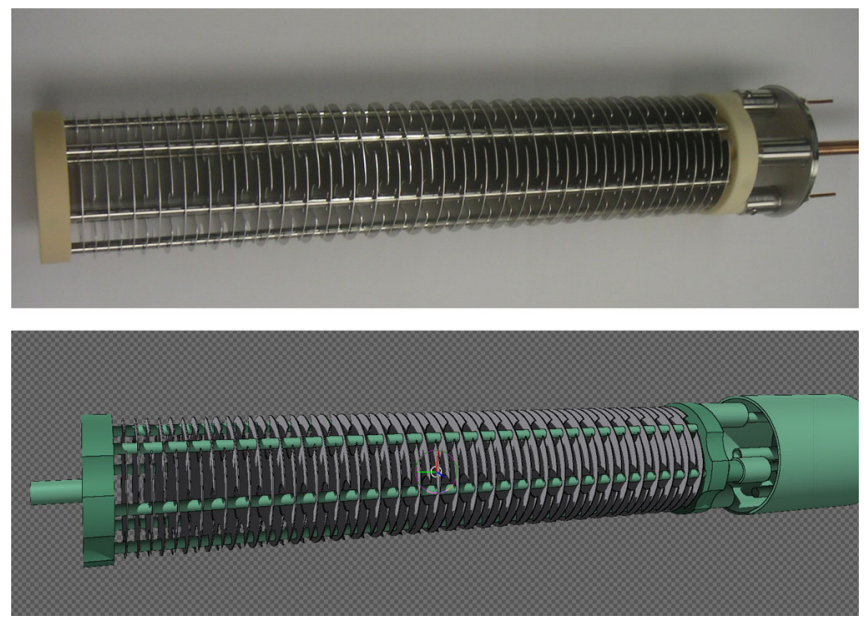

FIG. 2. Interior of a standard LHC beam loss monitor (top) and the corresponding FLUKA model used in the particle shower simulations (bottom). The 61 aluminum electrodes are connected by means of stainless steel rods, which are supported by thick alumina plates on both ends of the chamber. 
The BLM geometry model used in the particle shower simulations accurately reproduces electrodes, rods, spacers, insulators, and the stainless steel housing (see Fig. 2). The BLM response is obtained by calculating the energy deposition in the cylindrical gas volume between the 61 BLM electrodes. This is a simplified approach, since charges can also be collected from radii larger than the electrode radius if they are released in the vicinity of signal electrodes, while not all charges between electrodes are collected if they are produced close to a high-voltage electrode. In addition, the electric field and, hence, the active volume are distorted by the rods connecting the highvoltage electrodes. Figure 3 shows a two-dimensional contour plot indicating the active volume in the vicinity of the signal and high-voltage electrodes as predicted by numerical simulations of the charge induction on signal electrodes. Integrated over the entire ionization chamber, the active volume differs by less than $0.5 \%$ from the simplified sensitive volume used in the particle shower simulations. The systematic error arising from the simplified volume shape is estimated to be at most a few percent. A similar method for calculating the BLM response has been used in a previous study [51], with the exception that the delimiting radius for the active volume was assumed to be a few millimeters larger than the electrode radius.

The relative contribution of hadronic and electromagnetic shower components to the energy deposition in the

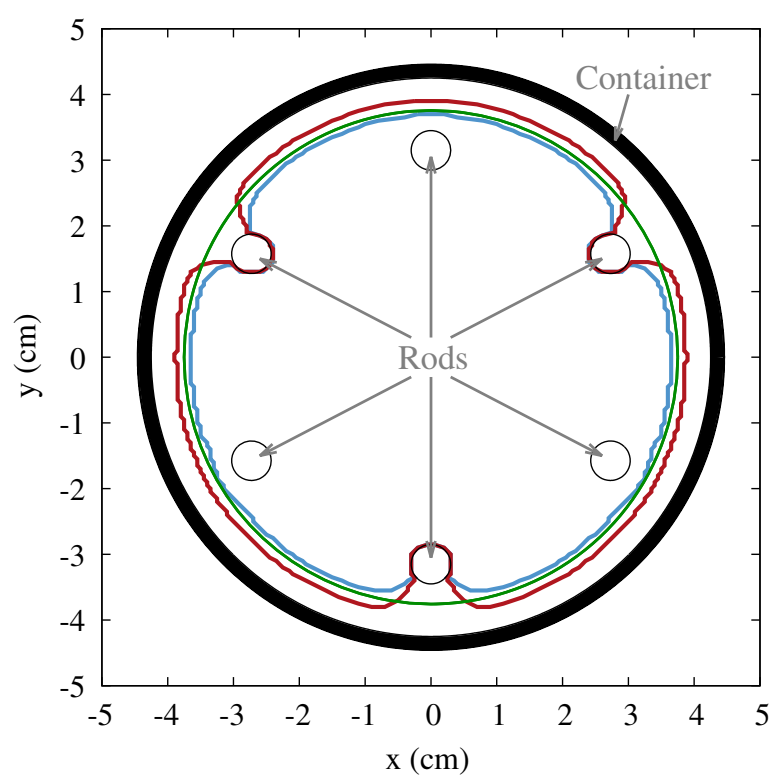

FIG. 3. Two-dimensional contour plot indicating the active BLM volume in the vicinity of high-voltage (blue line) and signal electrodes (red line). For comparison, the plot also delineates the electrode radius (green line), which is also the limit of the active volume assumed in the particle shower simulations. Results derive from numerical simulations of the charge induction on signal electrodes, based on the Green's function of electrons in the electric field created by electrodes, rods, and walls of the ionization chamber. active gas volume depends on the BLM position. Electromagnetic showers typically dominate signals at less shielded locations, for example, close to the beam pipe or magnet interconnects, whereas the relative contribution of charged hadrons and neutrons can be important for BLMs in the shadow of massive objects like magnets. In order to describe the peripheral shower leakage to BLMs, the production and transport of secondary particles were simulated down to low energies. The computational time is mainly governed by electron and positron transport. The transport thresholds were optimized separately for BLMs and other parts of the geometry model. Secondary electrons and positrons with a kinetic energy of less than a few $\mathrm{MeV}$ cannot penetrate the 2-mm-thick steel housing of BLMs, and, therefore, sub-MeV electrons and positrons outside of BLM chambers can be discarded without significantly affecting BLM signals. Photons from electron-positron annihilation are still produced when a positron falls below the threshold. These photons can, hence, contribute to the energy deposition in the BLM. The transport of photons outside of BLM chambers is terminated at $100 \mathrm{keV}$, as their contribution to BLM signals becomes small, although they can still penetrate the steel housing. In order to accurately simulate the electronic equilibrium between the nitrogen gas and chamber components like electrodes, spacers, and walls, it is necessary to explicitly transport sub-MeV electrons and positrons produced inside BLMs. Thresholds were therefore set to $10 \mathrm{keV}$, such that the range of electrons and positrons in the $\mathrm{N}_{2}$ gas is smaller than the distance between neighboring electrodes. The same thresholds were applied for photons inside BLMs. To probe the sensitivity of BLM signals to electron, positron, and photon transport settings, the thresholds inside BLMs were decreased to $1 \mathrm{keV}$ for a selected test case (wire scanner test shown in Sec. III A). For seven out of the eight BLMs included in the setup, the results agreed within the standard deviation of the two simulations, while for one BLM the results agreed within two standard deviations. A second sensitivity study was performed for transport thresholds outside of BLMs. As expected, no statistically significant differences were observed when reducing electron and positron thresholds from $1 \mathrm{MeV}$ to $100 \mathrm{keV}$ and photon thresholds from 100 to $10 \mathrm{keV}$. The chosen electron, positron, and photon transport settings are, therefore, considered suitable for the simulation benchmarks. Other particles (charged hadrons and muons) were transported down to $100 \mathrm{keV}$ in all regions of the geometry model, while neutrons were followed down to $10^{-5} \mathrm{eV}$. The impact of charged hadron and muon thresholds was probed using the same test case as above. No statistically significant differences were observed when reducing the transport thresholds to $10 \mathrm{keV}$.

A more important source of errors can be the assumed BLM position in the simulation model, in particular, for BLMs located close to the beam vacuum chamber where 


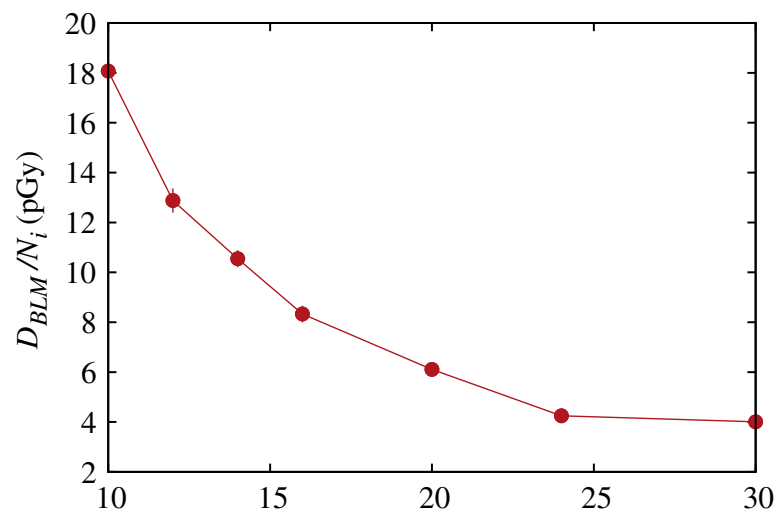

Distance between BLM and vacuum chamber $(\mathrm{cm})$

FIG. 4. Example of the simulated BLM response as a function of the radial distance between the vacuum chamber and BLM (center to center). The chamber and monitor are oriented parallel to each other. As source term, we consider collisions of $3.5 \mathrm{TeV}$ protons with the LHC wire scanner (carbon fiber). For simplicity, the spatial loss distribution was assumed to be pointlike. Signals are expressed per inelastic proton-nucleus collision $N_{i}$ in the wire. Vertical bars indicate, where visible, the statistical error of simulation results.

radiation fields develop a strong radial gradient. Figure 4 illustrates the dependence of BLM signals on the radial distance between the monitor and beam pipe. The shown signals correspond to losses of $3.5 \mathrm{TeV}$ protons induced by the LHC wire scanner (see also Sec. III A). The wire scanner can be considered as an almost pointlike source of losses. The BLM considered in Fig. 4 is located next to a standard room-temperature drift chamber, approximately $53 \mathrm{~m}$ downstream of the loss location. In this particular case, a radial displacement of the monitor by a few centimeters can alter the monitor response by several tens of percent. In all simulation studies presented in this paper, BLMs were placed at their nominal position specified in the internal CERN layout database or according to the best of our knowledge. For some BLMs, it cannot be excluded that the systematic uncertainty due to a wrong positioning amounts to a few tens of percent. This concerns mainly BLMs in room-temperature regions, while BLMs mounted on cold magnets are less affected, since their distance from the beam is well defined by the cryostat dimension.

Other sources of errors are not unique to BLM response calculations but apply to particle shower studies, in general. These include uncertainties in the physics models, approximations of geometric details and material compositions, and uncertainties in the particle loss distribution. For example, the real accelerator aperture can differ from the ideal aperture because of manufacturing tolerances or a nonideal alignment, which, in turn, affects the loss location of particles. Considering tolerances on cold bore and beam screen diameters, tolerances on the beam screen positioning, etc., the real aperture can differ by about one millimeter from the nominal beam screen radius. For impact angles of a few hundred microradians, this means that the actual loss location can differ by several meters from the nominal one. Such differences between expected and nominal loss location have, for example, been observed for dispersive $\mathrm{Pb}$ losses in the LHC dispersion suppressors. In this case, the discrepancy was clearly visible on BLMs, as the ion impacts on the beam screen were very localized (within a few meters). In other scenarios, the effect of aperture imperfections on BLM signals can be less obvious, for example, in the case of diluted losses over longer distances. In such cases, the effect of local aperture imperfections on BLM signals can be washed out by secondary showers created at different impact locations. The systematic uncertainty due to the absence of aperture imperfections in the simulation model is, hence, difficult to quantify and depends on the specific loss case. Local distortions of the particle loss distribution can also arise from short aperture discontinuities which are not included in the simulation model, for example, beam position monitors or vacuum valves. These discontinuities are generally shorter than $10 \mathrm{~cm}$. Considering the solid angle coverage of BLM chambers due to the chamber size and the distance from the beam pipe (typically a few tens of centimeters), it is estimated that the effect on BLM signals due to the absence of such discontinuities in the simulation model is limited. As for the aperture imperfections, it is, however, difficult to provide a general estimate of the simulation uncertainty, since this depends on the loss scenario, the concerned accelerator region, and the BLM positions.

\section{CONTROLLED BEAM LOSS EXPERIMENTS}

Magnet quenches have a negative impact on the LHC performance, as it can take hours to recover from a quench. An accurate knowledge of quench levels is therefore essential for setting BLM thresholds which trigger a beam abort before a beam-induced quench occurs. Having a good understanding of quench levels is also important for identifying the need of hardware upgrades for future operation at higher intensities and luminosities. A series of beam loss experiments with proton beams at different energies was carried out in 2010-2015 to probe the quench level of superconducting magnets in the LHC long straight sections, dispersion suppressors, and arcs $[21,55,56]$. The experiments involved different methods to generate beam losses in a controlled way, for instance, by injecting and dumping bunches on a closed collimator, by performing slow speed scans with the wire scanner, by using a closed orbit bump with a dynamically increasing bump amplitude, or by blowing up the beam with the transverse feedback system.

The different tests were analyzed by means of extensive simulation studies which involved particle tracking and shower simulations, as well as electrothermal simulations for evaluating the quench behavior of magnet coils [21]. The experiments conversely offered the opportunity to 
validate energy deposition simulations for controlled loss conditions. In the following, a validation of BLM response calculations is presented for two of the experiments, one exploring pointlike losses with the wire scanner [57] and the other one studying halo impact on collimators in the betatron cleaning insertion [69]. Selected results of the energy deposition and BLM studies for these tests have been reported previously in Refs. [21,41,57,58]. Here we provide a much more detailed assessment of the simulation accuracy which was beyond the scope of the other papers.

\section{A. Beam losses induced by the wire scanner}

In 2010, the LHC wire scanner was used in a test campaign to artificially induce almost pointlike losses of $3.5 \mathrm{TeV}$ proton beams. In this case, almost pointlike means that the obstacle is much smaller than the inelastic nuclear interaction length of the beam protons, as elaborated below. The goal of the wire scanner test was to probe quench levels for millisecond loss durations. The test has been described in Refs. [21,57], but for completeness some details are repeated here. The LHC is equipped with eight wire scanners, two per beam and plane, which are located in the radio-frequency insertion region (IR4). Each wire scanner accommodates a 34- $\mu$ m-thick carbon fiber which moves with a constant velocity of $100 \mathrm{~cm} / \mathrm{s}$ through the beam. The wire scanners are installed $34 \mathrm{~m}$ upstream of a stand-alone cryogenic unit containing a superconducting separation dipole (D2) and an assembly of superconducting orbit corrector and quadrupole (Q4). The beam loss experiment was carried out using the anticlockwise rotating beam, with a stored beam intensity of $1.53 \times 10^{13}$ protons. The losses induced by the fiber were selectively increased from scan to scan by successively reducing the wire speed from 100 to $5 \mathrm{~cm} / \mathrm{s}$. During the last scan, the downstream separation dipole quenched.

A series of BLMs around the D2 and Q4 measured the energy deposition from secondary showers induced by the collision products from the fiber. The measured dose can be assumed to be proportional to the number of inelastic nuclear collisions $N_{i}$ of protons in the wire during a scan. Elastically scattered protons do not contribute to the energy deposition in neighboring magnets and BLMs, as they typically remain in the beam envelope or they are lost in the betatron cleaning insertion. Assuming the wire moves with constant speed $v_{w}$ through the beam, the number of inelastic proton-nucleus collisions in the wire can be expressed as

$$
N_{i}=N_{b} N_{p} \frac{f_{r} d_{w}}{v_{w}} p_{i}
$$

where $N_{b}$ is the number of bunches, $N_{p}$ is the number of protons per bunch, $f_{r}$ is the LHC revolution frequency $(11245 \mathrm{~Hz}), d_{w}$ is the wire diameter, and $p_{i}$ is the probability that a proton traversing the wire has an inelastic interaction. Equation (1) holds independently of the actual transverse shape of the beam. The inelastic collision probability is given by

$$
p_{i}=1-\exp \left(-d_{a v} / \lambda_{i}\right),
$$

where $d_{a v}$ is the average path length of beam protons inside the wire and $\lambda_{i}$ is the inelastic nuclear mean free path in carbon. Assuming for simplicity that all protons have the same direction perpendicular to the scanning plane, the average path length in a round wire can be written as

$$
d_{a v}=\frac{d_{w} \pi}{4},
$$

which yields $26.7 \mu \mathrm{m}$ for the considered wire diameter of $34 \mu \mathrm{m}$. The inelastic nuclear mean free path in the wire is given by

$$
\lambda_{i}=\frac{M}{\rho_{w} N_{A} \sigma_{i}},
$$

where $M$ is the molar mass of carbon, $\rho_{w}$ is the wire density, $N_{A}$ is the Avogadro constant $\left(6.02214086 \times 10^{23} \mathrm{~mol}^{-1}\right)$, and $\sigma_{i}$ is the inelastic nuclear cross section. The latter is $258 \mathrm{mb}$ for inelastic collisions between $3.5 \mathrm{TeV}$ protons and carbon nuclei at rest (value taken from FLUKA). The wire density was measured to be $1.63 \mathrm{~g} / \mathrm{cm}^{3}$, with a standard deviation of $0.08 \mathrm{~g} / \mathrm{cm}^{3}$ [70]. The resulting mean free path $\lambda_{i}(47.6 \mathrm{~cm})$ is much larger than $d_{a v}$, and, hence, the interaction probability in Eq. (2) can be approximated as

$$
p_{i} \approx \frac{d_{a v}}{\lambda_{i}}=\frac{d_{w} \pi \rho_{w} N_{A} \sigma_{i}}{4 M} .
$$

Inserting Eq. (5) into (1), the number of collision becomes

$$
N_{i}=N_{b} N_{p} \frac{f_{r} d_{w}^{2} \pi \rho_{w} N_{A} \sigma_{i}}{4 v_{w} M} .
$$

This shows that $N_{i}$ depends quadratically on the wire diameter, and therefore a good knowledge of the wire properties is important for the benchmark. For a beam intensity of $1.53 \times 10^{13}$ protons, Eq. (6) predicts between $3.3 \times 10^{8}\left(v_{w}=100 \mathrm{~cm} / \mathrm{s}\right)$ and $6.6 \times 10^{9}\left(v_{w}=5 \mathrm{~cm} / \mathrm{s}\right)$ inelastic nuclear encounters per scan. The decay of beam intensity due to beam halo losses at collimators was about $1 \%$ between the first and the last scans and is neglected here.

The BLM signals measured during different scans showed some deviation from the ideal dependence on $v_{w}^{-1}$ expected from Eq. (6). This is illustrated in Fig. 5, which displays the ratio of the dose in the first BLM downstream of the wire scanner to the number of interactions predicted by Eq. (6). Signals measured by other 


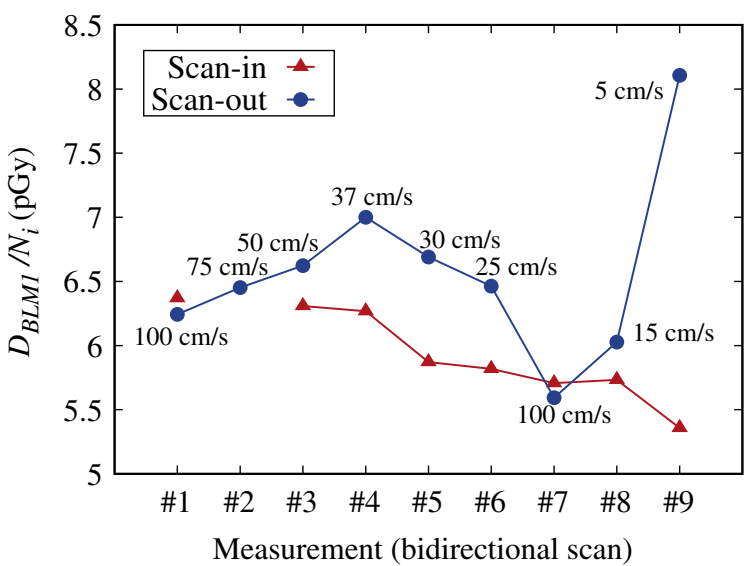

FIG. 5. Measured BLM dose divided by the number of inelastic nuclear collisions of protons in the LHC wire scanner predicted by Eq. (6). Each measurement consisted of a bidirectional scan, represented by separate points for the opposite scanning directions. The wire speed was always $100 \mathrm{~cm} / \mathrm{s}$ during the first of the two scans (triangles), while the second scan (circles) was performed at different speeds as indicated in the figure. For one of the scans (scan in for measurement number 2), no BLM data are available.

BLMs exhibited a similar variation. The observed behavior can be attributed to wire vibrations and to sublimation of the wire material due to excessive heating during lowerspeed scans [21,57]. Wire damage induced by particle beams has been observed previously in the CERN Super Proton Synchrotron [71]. A wire with a reduced diameter $d_{w}^{r}$ leads to a factor $\left(d_{w} / d_{w}^{r}\right)^{2}$ less inelastic collisions than an undamaged wire, while vibrations can either enhance or decrease the prediction of Eq. (6) depending on the interplay between the wire speed, beam size and amplitude, frequency, and phase of the oscillations. The presence of such vibrations together with the observed wire damage makes it difficult to reliably estimate the number of inelastic proton-nucleus collisions for these scans. In the following comparison, we therefore restrict ourselves to the first four scans at $v_{w}=100 \mathrm{~cm} / \mathrm{s}$, for which BLM signals varied by less than $3 \%$ between scans. Any scan where the wire is believed to have suffered prior damage is omitted.

As a source term for the shower simulations, inelastic proton-nucleus collisions were sampled according to a Gaussian transverse beam distribution at the position of the wire. The probability that the collision products interact hadronically inside the wire is very small and was neglected. The normalized emittance was assumed to be $2.5 \mu \mathrm{mrad}$ as measured by the wire scanner in the horizontal plane. Since no vertical scan was performed, the same normalized emittance was assumed in the vertical plane. This emittance corresponds to a beam size of $\sigma_{x}=$ $270 \mu \mathrm{m}$ and $\sigma_{y}=490 \mu \mathrm{m}$ at the wire scanner. The synchrotron light monitors showed some vertical emittance blowup during the fill, which was neglected in the simulations. The beam orbit at the wire scanner was

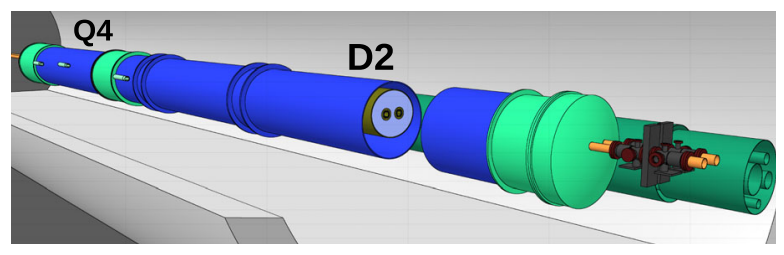

FIG. 6. Geometry model of the cryogenic unit downstream of the wire scanner, containing the D2 dipole and the Q4 quadrupole. The cryostat and the dipole are cut open to show the interior.

assumed to be at its nominal position. Previous measurements have shown that BLM signals at the Q4 and D2 are not very sensitive to a transverse displacement of the beam at the wire scanner. In these tests, the beam was displaced by up to $4 \mathrm{~mm}$ from its nominal position. This suggests that the above assumptions about beam position and vertical beam size suffice for the benchmark study.

Figure 6 displays the geometry model used in the shower simulations. The production of secondary particles was simulated by means of the PEANUT model in FLUKA. Figure 7 shows an absolute comparison of simulated and measured signals along the D2 and Q4. The dose values are displayed as a function of the $s$ position of BLMs with respect to IP1. As in Fig. 5, measurements are normalized according to the number of collisions predicted by Eq. (6). All BLMs considered in the figure are mounted on the magnet cryostats, with the exception of the last BLM, which is located in the proximity of the beam pipe downstream of the Q4. For clarity, the figure contains a schematic view of magnets (gray boxes) and BLMs

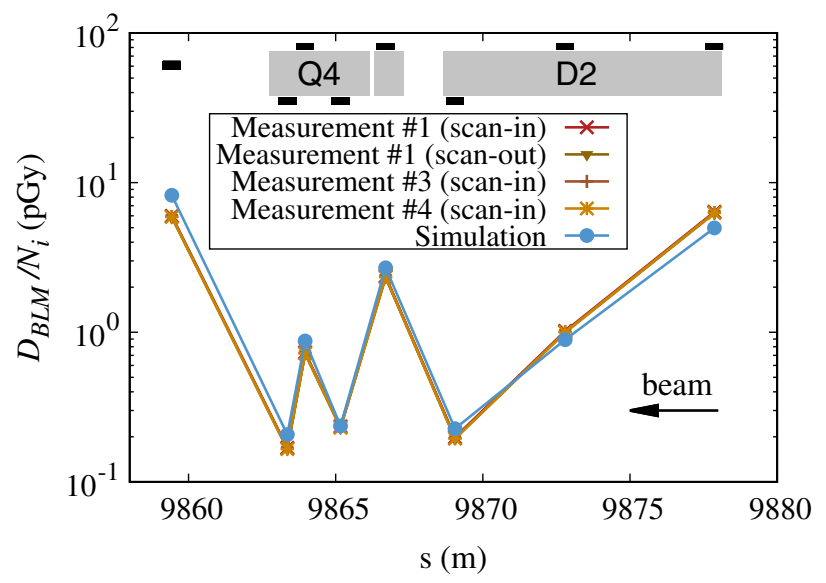

FIG. 7. Simulated and measured BLM signals for proton losses induced by the LHC wire scanner. The measurements derive from a controlled beam loss experiment carried out at $3.5 \mathrm{TeV}$ (see also Fig. 5). The beam direction is from the right to the left. The wire scanner is located at $s=9912 \mathrm{~m}$, approximately $34 \mathrm{~m}$ upstream of the first BLM on the D2 cryostat. All dose values are given per inelastic proton-nucleus collision in the wire. The statistical error of simulation results is less than $6 \%$. The data points are connected by lines to guide the eye. 
(black boxes). Similar illustrations are adopted in other sections of the paper.

The simulation results reproduce well the large variation of measured signals; in particular, they accurately describe the attenuation of particle showers in magnets and the leakage of secondary particles from the D2-Q4 interconnect. The interconnect provides only little shielding, and, hence, one can observe an elevated signal in the first BLM downstream of the interconnect. Conversely, the strongest attenuation of showers and, therefore, the smallest signals can be observed for BLMs which are installed on the side of the counterrotating beam. For all BLMs, the agreement between the simulation and measurement is better than $40 \%$. On average, the simulations are about $10 \%$ higher than the measurements. The discrepancies for individual BLMs could be due to approximations in the geometry model. Considering the very good overall agreement, the simulation results were used in our previous publications $[21,57]$ to empirically determine the number of protons lost during the last scan resulting in the D2 quench. This was done by scaling the simulated BLM pattern in Fig. 7 such that the best agreement with the measured pattern at $5 \mathrm{~cm} / \mathrm{s}$ was achieved.

\section{B. Collimation losses}

The LHC accommodates a multistage collimation system consisting of separate collimator hierarchies for momentum and betatron cleaning [6,7]. Each hierarchy comprises primary and secondary collimators made of carbon fiber reinforced carbon, which are complemented by movable tungsten-based absorbers and fixed-aperture masks. The cleaning systems are installed in dedicated insertion regions (IR3 and IR7) and are complemented by tungsten-based tertiary collimators at $145 \mathrm{~m}$ from IP1 (ATLAS) and IP5 (CMS) and $115 \mathrm{~m}$ from IP2 (ALICE) and IP8 ( $\mathrm{LHCb})$. In total, the two rings accommodate more than 100 collimators and absorbers. The multistage systems have so far demonstrated an excellent efficiency in removing unwanted halo particles from the beams [37]. A potential performance limitation, however, arises from off-momentum protons escaping the betatron cleaning insertion. Most of these protons originate from singlediffractive interactions in the primary collimators and are lost in the neighboring dispersion suppressors due to the elevated dispersion function. Several beam loss experiments with 3.5, 4, and $6.5 \mathrm{TeV}$ proton beams were carried out in 2011-2015 in order to estimate the risk of magnet quenches during periods of a short beam lifetime $[21,69,72,73]$. In these tests, which also served as a means to assess the overall collimation performance in case of very high losses, the particle loss rate in the collimation system and, hence, the leakage to the dispersion suppressor were deliberately increased by crossing a third-order resonance or by exciting the beam with the transverse feedback kicker.
In this section, we benchmark energy deposition simulations against BLM measurements recorded during the quench test at $4 \mathrm{TeV}$. The test, which has been described in more detail in Refs. [21,69], was carried out in 2013 using the anticlockwise circulating beam. The beam emittance was increased in a controlled way by means of white noise excitation, which generated steadily increasing losses for a duration of several seconds. To enhance the power deposition in superconducting magnets, larger collimator gaps were used than in standard $4 \mathrm{TeV}$ operation. Three attempts were made in separate fills to induce a magnet quench. In the last fill, a peak loss rate of about $1.6 \times 10^{12}$ protons per second was achieved, corresponding to a peak power loss of more than $1 \mathrm{MW}$. The BLMs showed clear evidence of particle leakage to the dispersion suppressor and the adjacent arc cells, but no quench was observed.

The benchmark study in this paper includes measurements from both the collimation insertion and the neighboring cold section. The simulation setup extends over almost $800 \mathrm{~m}$ of beam line and includes more than 100 BLMs. The quench test offers ideal conditions for benchmarking energy deposition simulations for betatron collimation losses. The presence of only one beam, together with the losses induced in one plane, leaves no ambiguity about the source term, whereas in regular collider operation BLMs are also exposed to showers generated by the counterrotating beam and losses could occur in both transverse planes. Another advantage is that the number of protons intercepted in the betatron cleaning insertion is given by the intensity loss measured by the beam current transformers. In regular operation, a non-negligible fraction of protons is also lost in the momentum cleaning insertion, and the sharing of betatron and momentum losses is not measured directly. A third advantage is the improved signal-to-noise ratio of BLM signals at cold magnets. Signals in the dispersion suppressor and the first arc cells are orders of magnitude smaller than in the insertion region. The high loss rate and relaxed collimator settings enhance the signal amplitudes at some magnets which would otherwise be dominated by noise.

The shower simulations in this section distinguish themselves from other studies in this paper through the complexity of the source term. A fraction of halo particles can traverse collimators multiple times in consecutive turns until they are eventually removed from the beam. Each time a particle traverses a collimator, it is subject to Coulomb scattering and loses energy through ionization and excitation of target atoms or through bremsstrahlung. In addition, particles can be subject to nuclear elastic and diffractive scattering. These processes alter the angle, position, and energy of a halo particle, which, in turn, influence its trajectory in subsequent turns. To get a full account of the scattering in collimators and the particle dynamics in the accelerator, we used coupled FLUKA-SIXTRACK simulations [74]. SixTrack [66] is a multiturn tracking code regularly 


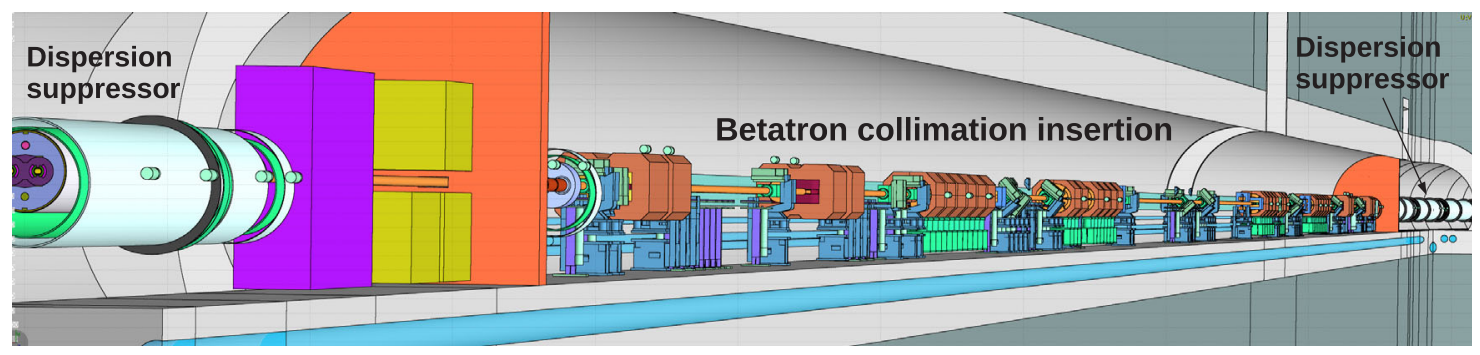

FIG. 8. Geometry model of the LHC betatron cleaning insertion and the first superconducting magnets in the dispersion suppressors.

used at CERN for evaluating the performance of the collimation system [37]. In our simulation setup, SIXTRACK accounts for the propagation of beam particles in lattice elements, while FLUKA is used to treat particlematter interactions in collimators. Starting from an annular halo distribution, particles were tracked over hundreds of turns until they were subject to an inelastic nuclear collision in a collimator jaw or until they touched the aperture of a magnet. The phase space coordinates of protons impacting on the faces of collimator jaws were then used as the source distribution for the shower simulations.

The shower simulations were carried out with a standalone FLUKA model (see Fig. 8) comprising the betatron cleaning insertion, the dispersion suppressor, and two adjacent arc half-cells. The model contained a full threedimensional description of the beam line and tunnel, whereas the coupled tracking-FLUKA simulation incorporated only models of collimators. Since only a small fraction of particles leaks to superconducting magnets, separate shower simulations were carried out for roomtemperature and cold accelerator regions. By decoupling these simulations, one can achieve a faster statistical convergence in the latter case by suppressing showers in upstream cells. The two different studies are discussed in the following subsections.

A similar simulation chain consisting of tracking and a shower simulation has been adopted previously in Ref. [37], which presented a first validation of magnet-to-collimator signal ratios against measurements from 2011. The simulation setup in the present paper is an evolution of the one used in Ref. [37], where not FLUKA but empirical interaction models embedded in SixTrack were used for simulating particle-collimator interactions in the first simulation step. Another difference concerns the description of collimation losses. In Ref. [37], the output of the first step and input for the second step was the phase space distribution of inelastic proton-nucleus collision points in collimators. In the present setup, the second step starts from the impact distribution of protons on the collimator surface as described above. This means that the loss points of beam protons inside the collimators are determined by FLUKA and not by the empirical SixTRACK models as in Ref. [37]. More details about the coupling between the FLUKA and SIXTRACK codes can be found in Ref. [58].

\section{BLM signals in the betatron cleaning insertion}

Figure 9 shows simulated and measured BLM signals in the region between the primary collimators, called TCPs, on the right side of IR7, and the tungsten absorbers (TCLAs) on the left side of IR7. The secondary collimators, called TCSGs, are located in between TCPs and TCLAs. The positions of absorbers and collimators installed on the anticlockwise circulating beam are indicated in the top part of the graphs. The separation dipoles (D3 and D4) and two out of the three matching section quadrupoles installed on each side of the IR (Q4 and Q5) are normal conducting, since the radiation levels would be too high for superconducting magnets. The BLM signals are expressed per proton intercepted by IR7 collimators and eventually lost in the machine $\left(N_{p}\right)$. Most of the protons are lost inside the collimators themselves, but a small fraction are lost somewhere else. The signal patterns show elevated signals downstream of collimators and absorbers because of particle showers escaping the absorber blocks. The openings of collimators and shower absorbers follow a hierarchy. During the test, the TCP jaws were placed $6.1 \sigma$ from the beam, the TCSG jaws were at $10.1 \sigma$, and the TCLA jaws were at $18.9 \sigma$ (all $\sigma$ values correspond to a normalized emittance of $3.5 \mu \mathrm{m} \mathrm{rad}$ ). The highest BLM signals occur right after the TCPs, while the signals at the TCLAs are about 2 orders of magnitude lower.

A first comparison, using the simulation data from Fig. 9, has been shown in Refs. [41,58], including also a comparison against the previously used method, where inelastic collision points in the collimators were obtained with the empirical models in SixTrack. This comparison showed that both simulation methods yield similar BLM results. This finding was not surprising, since the main difference between the two methods is the spatial distribution of the collision points, while in both cases FLUKA is used as the event generator for sampling inelastic collision products. The difference between the two methods becomes more appreciable when studying, for example, radiation damage or energy deposition inside the absorber blocks of the primary collimators. Since BLMs are located outside of the beam pipe, the BLM signals depend, however, much less on the spatial loss distribution inside the blocks.

In Refs. [41,58], only the measurement at the time of the quench was considered. In the present paper, the BLM 

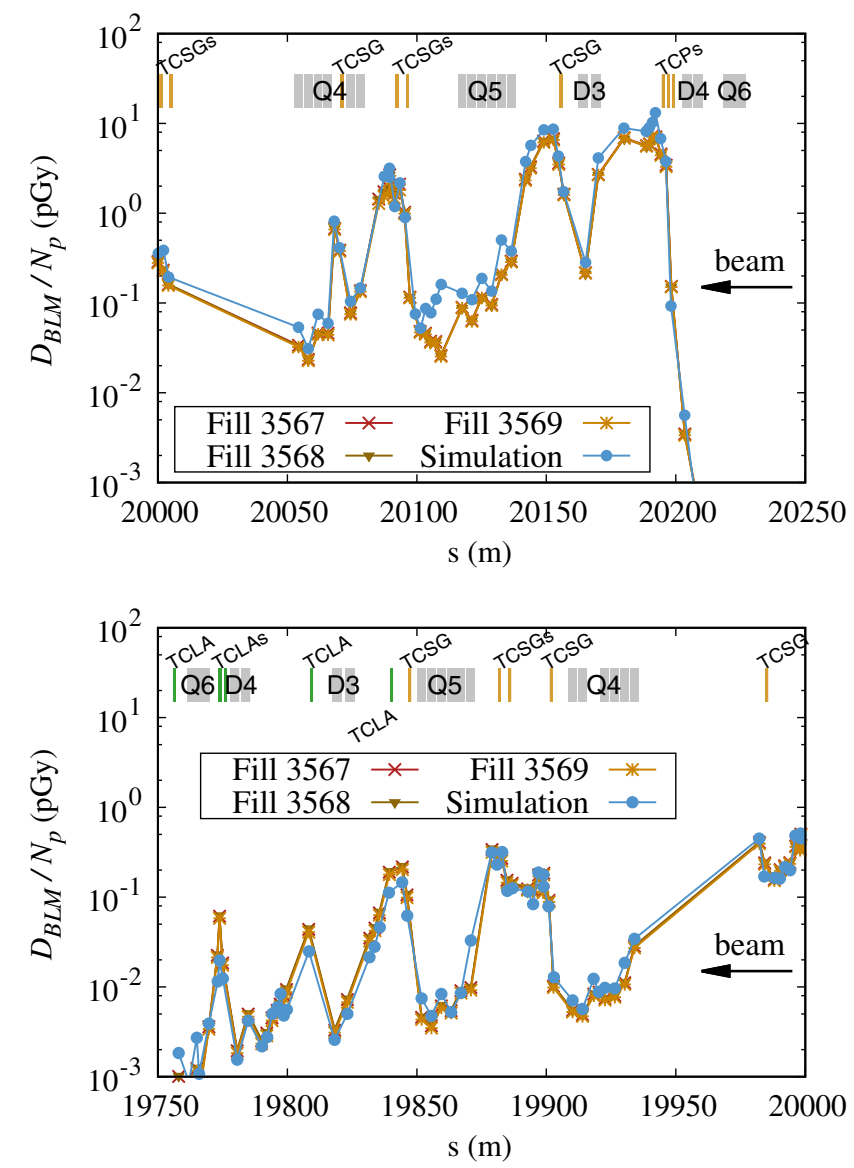

FIG. 9. Comparison of simulated and measured BLM signals induced by $4 \mathrm{TeV}$ proton losses in the betatron cleaning insertion. The top and bottom figures show the right and left side of the insertion region, respectively. The beam direction is from the right to the left. The positions of primary and secondary collimators (TCPs and TCSGs), tungsten absorbers (TCLAs), dipoles (D3 and D4), and quadrupoles (Q4-Q6) are illustrated in the upper part of the graphs. All signals are expressed per proton lost in the collimation system. The statistical error of simulation results is generally smaller than $10 \%$ for dose values above $\sim 10^{-2}$ pGy but can be as large as a factor of a few for dose values below $10^{-2} \mathrm{pGy}$. The measurement are labeled by the corresponding LHC fill number, which is an unique identifier for a LHC fill.

response is averaged over a longer time interval, which reduces significantly the measurement uncertainty. The different measurements, which are identified in Fig. 9 by the respective LHC fill number, correspond to the different attempts of quenching a magnet. For each fill, the measured signals were time integrated over the entire loss duration (between 5 and $13 \mathrm{~s}$ ) and were normalized to the intensity loss recorded by the beam current transformers (between $2 \times 10^{12}$ and $9 \times 10^{12}$ protons). The time-integrated dose values were corrected for the noise floor, which was determined individually for each BLM by integrating the signal during a reference period without a beam between the fills. The normalized noise-corrected measurements from the different fills agree within $4 \%$ in the region of the TCPs but exhibit somewhat larger discrepancies of up to $18 \%$ around other collimators and absorbers.

The measured patterns are generally well reproduced by the simulation. For more than $90 \%$ of all BLMs, measured and simulated signals agree within a factor of 2 , and in almost half of all cases the agreement is better than $30 \%$. The simulations show a slight tendency to overestimate measured signals at the TCPs, while the opposite is true for BLMs around the TCLAs. The overall agreement can still be considered as remarkable given the complexity of the simulation setup, the relatively large geometry model, and the important variation of BLM signals along hundreds of meters of beam line. A similar degree of agreement was obtained for a later quench test at $6.5 \mathrm{TeV}$, which was carried out in 2015. A first BLM comparison for this test has been presented in Ref. [43] but will be detailed in a future publication.

\section{BLM signals along the continuous arc cryostat}

A few per mille of protons undergoing a nuclear collision in the collimators escape the beam cleaning system and are lost in superconducting magnets located in the continuous arc cryostat. The main contribution is due to protons subject to single-diffractive scattering in the TCPs. The continuous cryostat starts about $24 \mathrm{~m}$ downstream of the last TCLA. The first superconducting magnet in the cryostat is a matching section quadrupole in cell 7, which is followed by a string of dispersion suppressor magnets (half-cells 8-11) and arc magnets (half-cells $\geq 12$ ). To achieve a faster statistical convergence of BLM calculations in the cold sector, the shower simulations were split in two parts. Starting from the precalculated impact distribution on collimators, only high-energy particles $(\geq 1 \mathrm{TeV})$ emerging from collimators or from consecutive showers were transported to the last TCLA and beyond, while sub-TeV particles were discarded. This allowed to reduce the original phase space distribution to a much smaller subset relevant for the cold section. In a second step, secondary showers initiated by the TeV particles in the TCLA and in downstream magnets were simulated down to low energies. The TCLA was included in the cold section simulations, since showers escaping from its jaws can contribute to the energy deposition in the first few magnets.

Figure 10 compares the obtained BLM signals with measurement data from the three different fills of the quench test. The locations of quadrupoles (Q7-Q13) and main dipoles (MBs) are illustrated in the top part of the graphs. The four dispersion suppressor half-cells comprise each two dipoles and a quadrupole, whereas a standard arc half-cell consists of three dipoles and a quadrupole. The last dispersion suppressor half-cell (cell 11) houses in addition a 13-m-long connection cryostat (LE), which is installed at the location of the missing dipole and ensures the continuity of electrical, cryogenic, and other systems. All the 

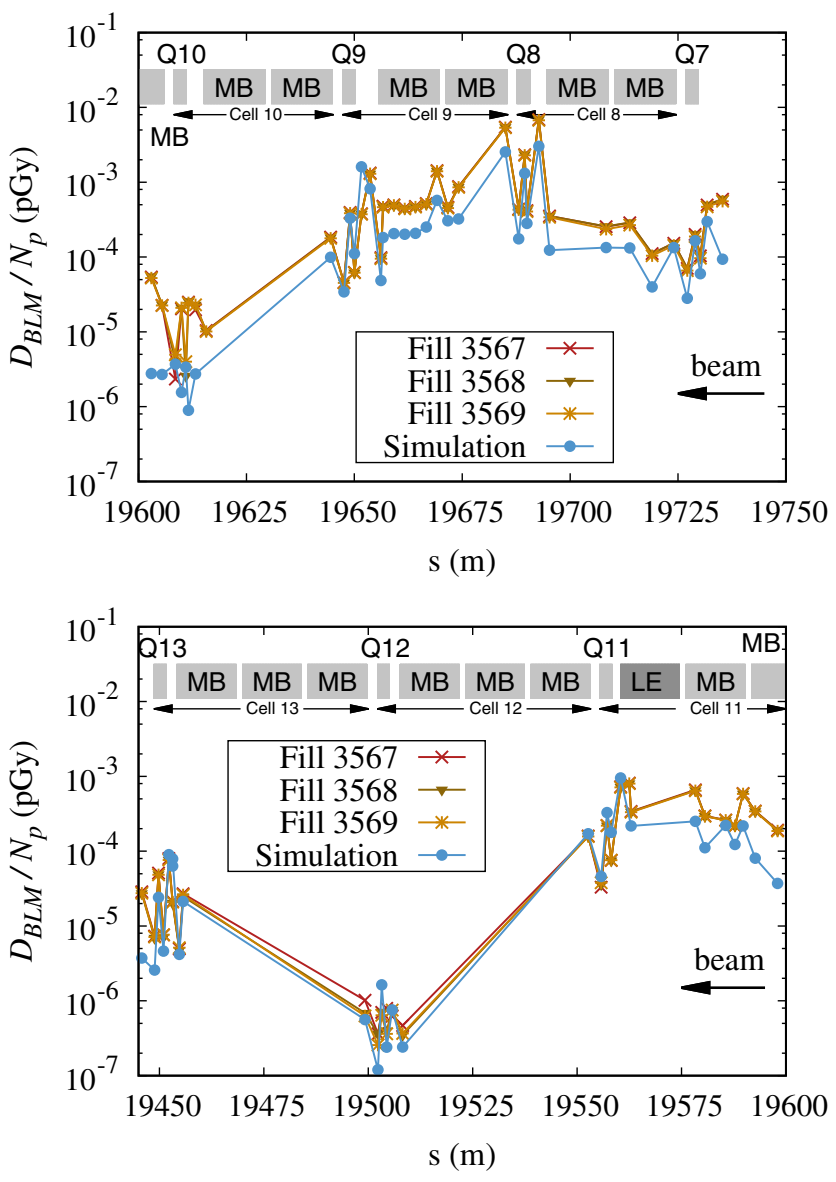

FIG. 10. Comparison of simulated and measured BLM signals in the dispersion suppressor (cells 8-11) and the first two arc half-cells (cells 12 and 13) next to the betatron cleaning insertion. The beam direction is from the right to the left. The measurements derive from the same fills as in Fig. 9. All signals are expressed per proton lost in the collimation system. The statistical error of simulation results is less than $10 \%$ for dose values above $\sim 10^{-4}$ pGy but can be as large as a factor of a few for dose values below $\sim 10^{-4} \mathrm{pGy}$.

quadrupoles, as well as the dipoles in cells 8,9 , and 11, are equipped with BLMs. The BLMs on dipoles are all located on the side of the anticlockwise rotating beam, with the specific purpose to detect leakage from the collimation insertion. Quadrupoles are equipped with BLMs on either side of the magnets.

Like in Sec. III B 1, the measured dose values were integrated over the entire loss duration, were corrected for the noise pedestal, and were normalized to the intensity loss measured by the beam current transformer. The data from the different fills agree within 10\%, except for some BLMs around the Q10 and the Q12, where signals remained close to the noise floor and the fill-to-fill variation is as high as a factor of 2.5.

The loss location of single-diffractive protons is closely correlated with the relative momentum loss the protons suffered in the scattering event. The losses are mainly concentrated in the half-cells 8,9 , and 11 , where they form two distinct loss clusters [37]. This is also reflected in the BLM pattern. The highest signals can be observed around the Q8. The simulation reproduces well the overall BLM pattern but systematically underestimates, by about a factor of 3, signals up to cell 11. A similar discrepancy was found in the previous benchmark study against 2011 measurements [37]. This study was, however, restricted to a few BLMs and presented only a relative comparison with respect to the signals at the TCPs. The same underestimation was observed for the later quench test at $6.5 \mathrm{TeV}$, as reported in Ref. [43]. Considering the otherwise good agreement in the upstream collimation region, the results suggest that the simulation underestimates the number of protons leaking to this part of the dispersion suppressor. The most probable cause for this underestimation is the presence of imperfections, such as possible tilts of collimator jaws with respect to the beam orbit, beam center errors due to orbit shifts and due to the achievable collimator alignment precision, collimator gap errors due to optics errors, and aperture misalignments. These imperfections, including a possible deviation from the ideal beam orbit, are not accounted for in the simulations. Previous tracking studies [37] demonstrated that such imperfections can increase the losses in cells $8-11$ by about a factor of 2 .

The simulation data from Fig. 10 have been used in our previous publication [21] to estimate the uncertainty of the energy deposition in the magnets with the highest power load. A detailed comparison as presented in this section was, however, beyond the scope of this paper.

Around the Q11, Q12, and Q13, the agreement between the simulation and measurement is better, which indicates that the number of protons leaking to this region is less affected by imperfections. Some of the measurements, in particular around the Q12, are subject to larger uncertainties, since the measured dose was close to the noise level. Together with the higher statistical error of simulation results, this can explain the differences observed for some of the BLMs. The systematic underestimation observed upstream of the Q11 is, however, not present around these quadrupoles.

\section{BEAM LOSSES IN REGULAR COLLIDER OPERATION}

This section presents simulation benchmarks against BLM signals measured during regular physics fills in run I. The considered source terms are proton losses induced by dust particles as well as proton and heavy ion collisions in the LHC detectors. The different loss cases are characterized by distinct spatial and temporal BLM signatures. While dust particles lead to transient losses at arbitrary locations and at irregular times, luminosity losses give rise to steady-state BLM signals in the insertion regions and the neighboring dispersion suppressors. 
Because of these characteristics, BLM measurements can be uniquely associated with the respective source terms.

\section{A. Beam losses induced by dust particles}

Micrometer-sized dust particles liberated into the LHC beams represented so far a major source of transient beam losses during proton operation. Events attributed to dust particles have been observed since the first high-intensity beams in run I and since then gave rise to several tens of beam aborts $[8,10,12-15]$. The first magnet quenches induced by dust particles occurred in 2015 when the LHC operated for the first time close to its design energy $[13,14]$. Beam losses due to dust particles have been observed previously in other machines, particularly in electron storage rings, where they manifested themselves as a sudden and sometimes persistent lifetime degradation due to the trapping of charged dust particles in the vicinity of the beam core [75-81]. Dust trapping phenomena can, however, be excluded for positively charged beams like in the LHC owing to the repelling force experienced by the dust particle as it becomes ionized during its passage through the beam tails [82-84].

The events observed in run I occurred in different sections of the LHC rings, with a significant accumulation around the LHC injection kickers in IR2 and IR8 and in certain arc cells [8,10-12]. The dust particles in the kicker magnets have been identified as debris from the ceramic vacuum chambers [11], while the source and nature of macroparticles in the arcs have not yet been conclusively determined. The occurrence of dust particle events in the kickers was successfully mitigated in 2012 by adopting an improved cleaning procedure for the ceramic chambers [11], whereas the transient loss events in the arcs still persist in present operation [13-15]. In the LHC jargon, these macroparticles are referred to as "unidentified falling objects."

Proton collisions with dust particles falling into the LHC beams typically appear as a localized increase of BLM signals. The events have a characteristic duration of several tens to several hundred microseconds $[8,10,12]$. In this section, we present dust-particle-induced signal patterns measured during proton operation with $4 \mathrm{TeV}$ beams in 2012 and compare them against predictions from particle shower simulations. We treat only inelastic collisions of protons with nuclei of dust particles, since elastically scattered protons do not contribute to the energy deposition in nearby magnets. As the number of inelastic protonnucleus collisions is a priori unknown, only a relative comparison of measured and simulated BLM patterns is possible. The number of collisions depends, for example, on the elemental composition of the dust particle but also on the obstacle size and its trajectory, which can vary substantially from event to event. The trajectory is governed by the rate at which the dust particle is charged and eventually repelled from the beam [82-84]. As the size and motion of the dust particle are unknown, it is assumed that dust particles are static and pointlike. The impact on the BLM response is estimated to be minor. The assumptions about the dust particle composition are detailed in the following subsections.

\section{Dust particle events in the arcs}

Figure 11 compares simulation predictions with measured BLM signal patterns attributed to dust-particleinduced loss events in a standard arc half-cell (19R3). The cell is located in the arc sector between the momentum cleaning insertion and the rf insertion. The geometry model used in the simulations is the one shown in Fig. 1. All patterns are expressed as a fraction of the maximum signal. Measured signals were time integrated over the entire loss duration, which typically lasted for a few turns. The events were recorded during regular $4 \mathrm{TeV}$ proton fills and occurred either during the betatron squeeze or during stable collisions in the experiments. The events shown in Fig. 11 were among the ones which produced the highest BLM signal amplitudes in this cell in 2012. A standard arc halfcell like 19R3 consists of three dipoles (MBs) and a short straight section. A half-cell is usually equipped with three monitors per beam, two being installed next to the quadrupole (MQ) and one downstream of the MQ-MB interconnect. The considered cell, however, exhibited one of the highest rates of transient beam losses in the arcs in 2011 and has therefore been equipped with additional BLMs on the MB cryostats to study the spatial distribution of dust particles. Since the nature of the dust particles in arc cells like 19R3 is not yet fully understood, it was arbitrarily assumed that the dust particles are composed of carbon. One can nevertheless get a good approximation of the BLM response, as the energy deposition in accelerator equipment exhibits a moderate dependence on the actual dust particle composition. Simulations show that, if the dust particle were made of a heavy element like gold, the highest signals nearby the loss location would vary by a few tens of percent.

The measured patterns shown in Fig. 11 indicate that the losses occurred at different positions inside the dipoles. As the exact position of the dust particles is a priori unknown, the loss locations had to be determined by finding the best match between the simulation and measurement. This was done by performing simulations for different loss locations. Figure 11 shows only results which yielded the best agreement with the measurements. Considering the sensitivity of simulated BLM patterns to the assumed loss location, it is estimated that the location of dust particles can be determined with an accuracy of about one meter. In general, the simulations accurately reproduce the relative variation of signal amplitudes over 2 orders of magnitude, except for some BLMs where the measured signals were close to the noise level. The latter applies, in particular, to 

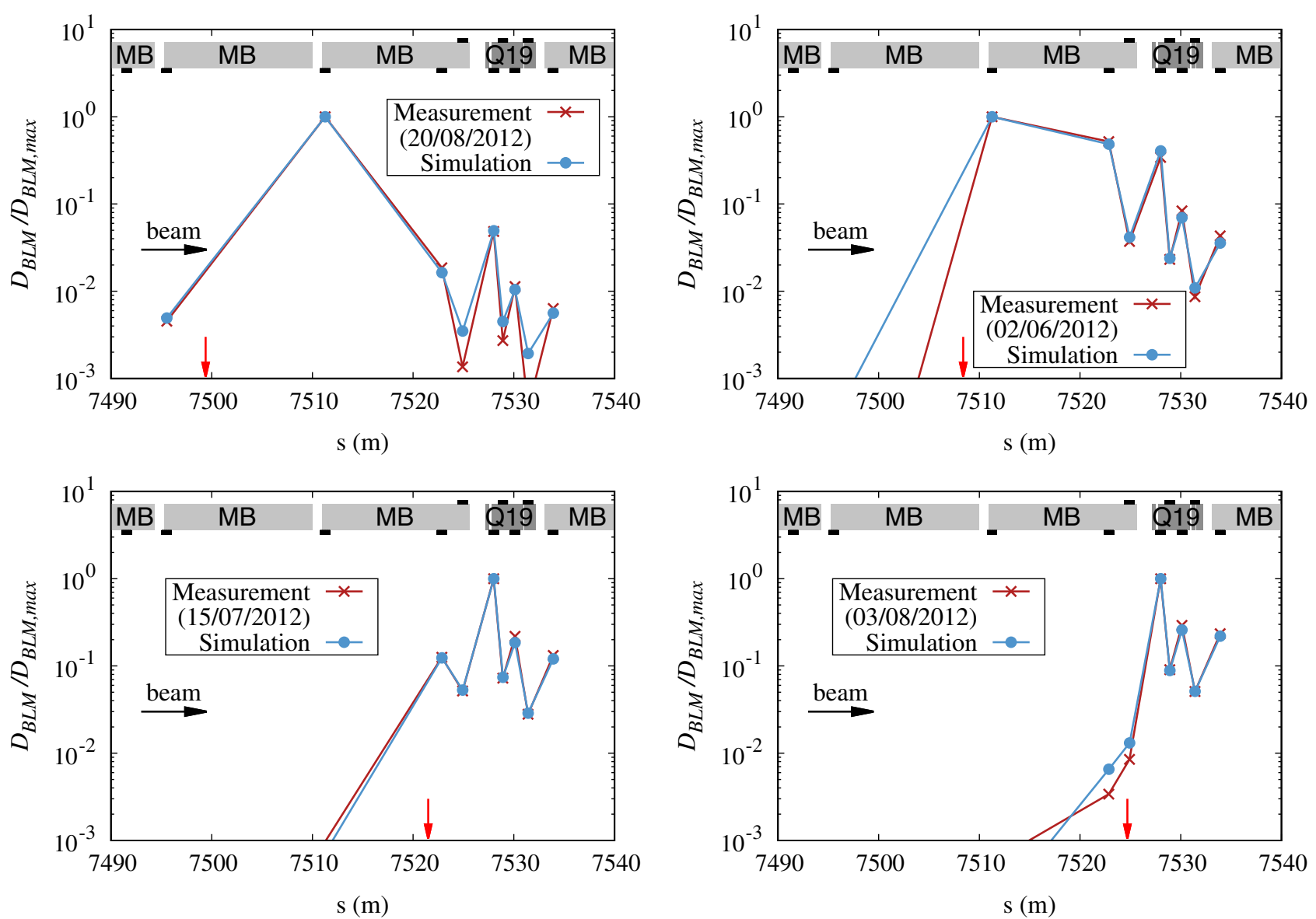

FIG. 11. Comparison of simulated BLM signals with measured BLM patterns attributed to dust-particle-induced beam losses in a standard LHC arc cell (19R3). All patterns are expressed as a fraction of the maximum signal. The beam direction is from the left to the right. The events occurred during regular $4 \mathrm{TeV}$ proton fills in 2012. The vertical arrows indicate the dust particle positions assumed in the simulations. The statistical error of the simulation results is less than $10 \%$, except for BLMs signals, which are less than $10^{-2}$ of the maximum signal.

BLMs upstream of the loss location, which measure the backscatter component of particle showers.

From the ratio of time-integrated measured signals and simulated signals, one can estimate that the number of inelastic proton-nucleus collisions was between $1 \times 10^{6}$ and $4 \times 10^{6}$ for the different events shown in Fig. 11. This is compatible with the fact that no change in beam intensity was measured by the beam current transformers, since such low-intensity losses are below their resolution.

\section{Dust particle events in the injection region}

Many of the dust events in run I occurred around the LHC injection kickers (MKIs). Figure 12 shows a FLUKA geometry model of the injection region in IR2, where the clockwise rotating beam is injected into the LHC. The four kicker modules are located upstream of a superconducting quadrupole (Q4) and separation dipole (D2). Figure 13 shows simulation results obtained with this model, together with measurements from a dust particle event in the MKIs in 2012. The event occurred during a regular proton fill at
$4 \mathrm{TeV}$, with a stored beam intensity of $2.2 \times 10^{14}$ protons. The BLMs triggered the extraction of the beams a few hundred microseconds after the onset of losses. Many of the transient loss events observed around the MKIs in 2011 and 2012 exhibited a similar BLM pattern as the one shown in the figure. As demonstrated in Refs. [11,36], where a first comparison between simulations and 2011

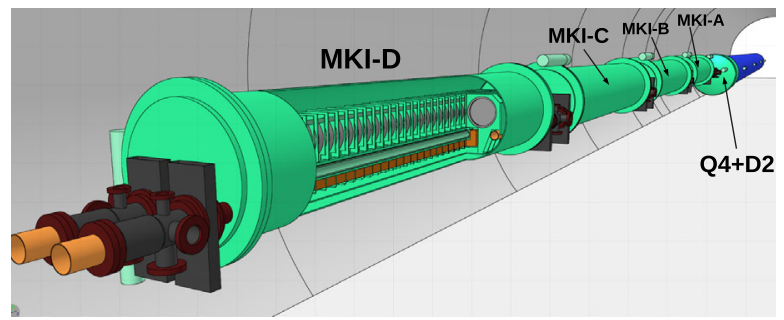

FIG. 12. Geometry model of the four injection kickers (MKIs) and the downstream cryounit, containing the D2 dipole and the Q4 quadrupole. The first kicker module (MKI-D) is cut open to show the interior. 


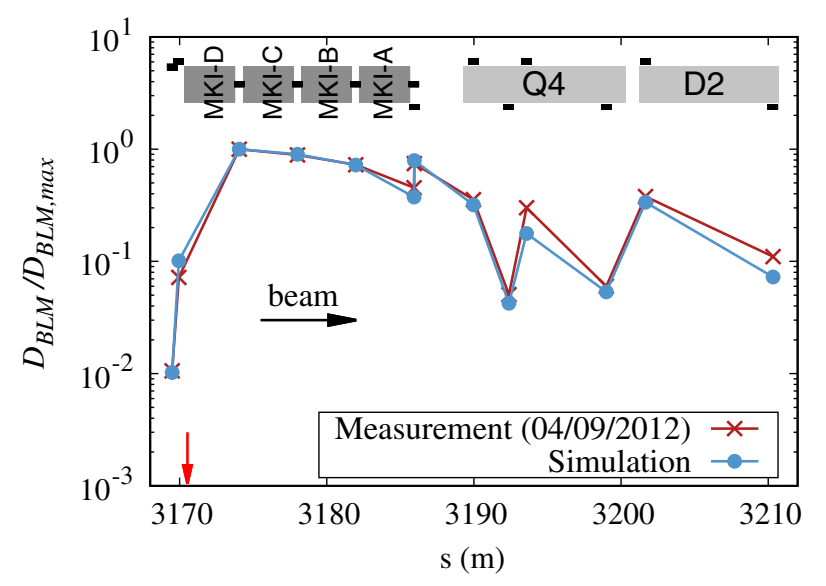

FIG. 13. Comparison of simulated and measured BLM signal patterns for a dust-particle-induced loss event in the LHC injection kickers in IR2. All patterns are expressed as a fraction of the maximum signal. The beam direction is from the left to the right. The event occurred during a fill with $4 \mathrm{TeV}$ protons in 2012. The vertical arrow indicates the dust particle position assumed in the simulation. The statistical error of the simulation results is less than $10 \%$, except for the two BLMs upstream of the assumed dust particle location.

measurements at $3.5 \mathrm{TeV}$ was presented, the BLM patterns indicated a locally increased dust contamination in the first of the four kicker modules (MKI-D in Fig. 13).

The kickers accommodate ceramic $\left(\mathrm{Al}_{2} \mathrm{O}_{3}\right)$ vacuum chambers which are equipped with screen conductor inserts to provide a passage for the beam image current. The observed loss events can likely be attributed to fragments which separated from the alumina tubes during assembly or manufacturing of the chambers [11]. In Fig. 13, we assumed that the beam interacts with an $\mathrm{Al}_{2} \mathrm{O}_{3}$ macroparticle at the upstream end of the MKI-D vacuum chamber. The simulation agrees well with the measured BLM signal pattern along the MKIs and the neighboring quadrupole and dipole. In particular, the large variation of signals, spanning 2 orders of magnitude, is well reproduced. The simulations suggest that approximately $9 \times 10^{8}$ protons had an inelastic encounter with the dust particle before the beams were dumped. Adopting the model described in Ref. [84], it is estimated that such beam losses can be caused by a $\mathrm{Al}_{2} \mathrm{O}_{3}$ macroparticle with a diameter of less than $30 \mu \mathrm{m}$. This is compatible with typical dust particle sizes found during the inspection of MKI chambers using a scanning electron microscope [11].

\section{B. Proton-proton collisions}

In run I, the LHC has delivered peak luminosities of up to $7.7 \times 10^{33} \mathrm{~cm}^{-2} \mathrm{~s}^{-1}$ to ATLAS and CMS, corresponding to an average of about 36 inelastic events per bunch crossing or to an inelastic collision rate of more than $0.5 \mathrm{GHz}$ [2]. The working points of the two other experiments were at lower luminosity, with $\mathrm{LHCb}$ operating around $4 \times 10^{32} \mathrm{~cm}^{-2} \mathrm{~s}^{-1}$ and ALICE around $1-3 \times 10^{30} \mathrm{~cm}^{-2} \mathrm{~s}^{-1}$. In 2016, the collision rate in ATLAS and CMS exceeded for the first time the design luminosity of $10^{34} \mathrm{~cm}^{-2} \mathrm{~s}^{-1}$ [85] and reached more than double this value in 2017 [5]. A very significant fraction of the energy released in the collisions is carried by secondary particles emitted in the forward direction, which can escape the experimental caverns and give rise to considerable heat deposition in neighboring final focus regions. The final focus quadrupole magnets are located $23 \mathrm{~m}$ from the IPs and consist of three cryoassemblies. Two of them host a single superconducting quadrupole of $6.37 \mathrm{~m}$ length (Q1 and Q3), while the central one combines two $5.5 \mathrm{~m}$ superconducting quadrupoles into a single functional entity (Q2). As particle collisions in the IPs represent the major source of radiation around these magnets, signals of nearby BLMs typically exhibit a good correlation with the instantaneous luminosity delivered to the experiments. This is illustrated in Fig. 14, which shows the time evolution of the luminosity in ATLAS and LHCb during a regular fill in 2012 together with the measured BLM dose rate at the Q1. While the luminosity delivered to ATLAS naturally decays throughout the fill, it is maintained at an almost constant value in LHCb by manipulating the transverse offset of the two counterrotating beams at the IP [86]. In both cases, BLM signals at the Q1 follow closely the luminosity evolution during the fill.

In this section, we compare BLM measurements in the final focus regions next to ATLAS and $\mathrm{LHCb}$ against simulation predictions of BLM signals for proton-proton collisions at a center-of-mass energy of $\sqrt{s}=8 \mathrm{TeV}$. The results for ATLAS have been shown previously in Ref. [40], however, without a deep discussion of the underlying models and methods. Another comparison, considering collisions at $\sqrt{s}=7 \mathrm{TeV}$ in CMS, was shown in Ref. [36]. In the present paper, we restrict ourselves to collision losses in the direction of the clockwise rotating beam. Similar results can, however, be expected for the other direction. The adopted simulation models included a detailed description of vacuum chambers, forward shielding, and absorbers in the experimental caverns. These elements intercept a significant fraction of the power released in the collisions and, therefore, determine the leakage to neighboring magnets. The geometry models are illustrated in Fig. 15. In the case of ATLAS, only the forward calorimeter and the relevant shielding and absorbers were included, while in the case of LHCb the entire detector was modeled. The absence of the detector in the first model (other than the forward calorimeter) is expected to have a negligible impact on the BLM signals, since the energy deposition in the triplet magnets is almost exclusively determined by collision products which remain within the geometrical acceptance of the secondary particle absorber, called TAS, which is located just upstream of the triplet magnets (see Fig. 15). These secondary particles carry about 56\% of the energy released in the proton-proton 

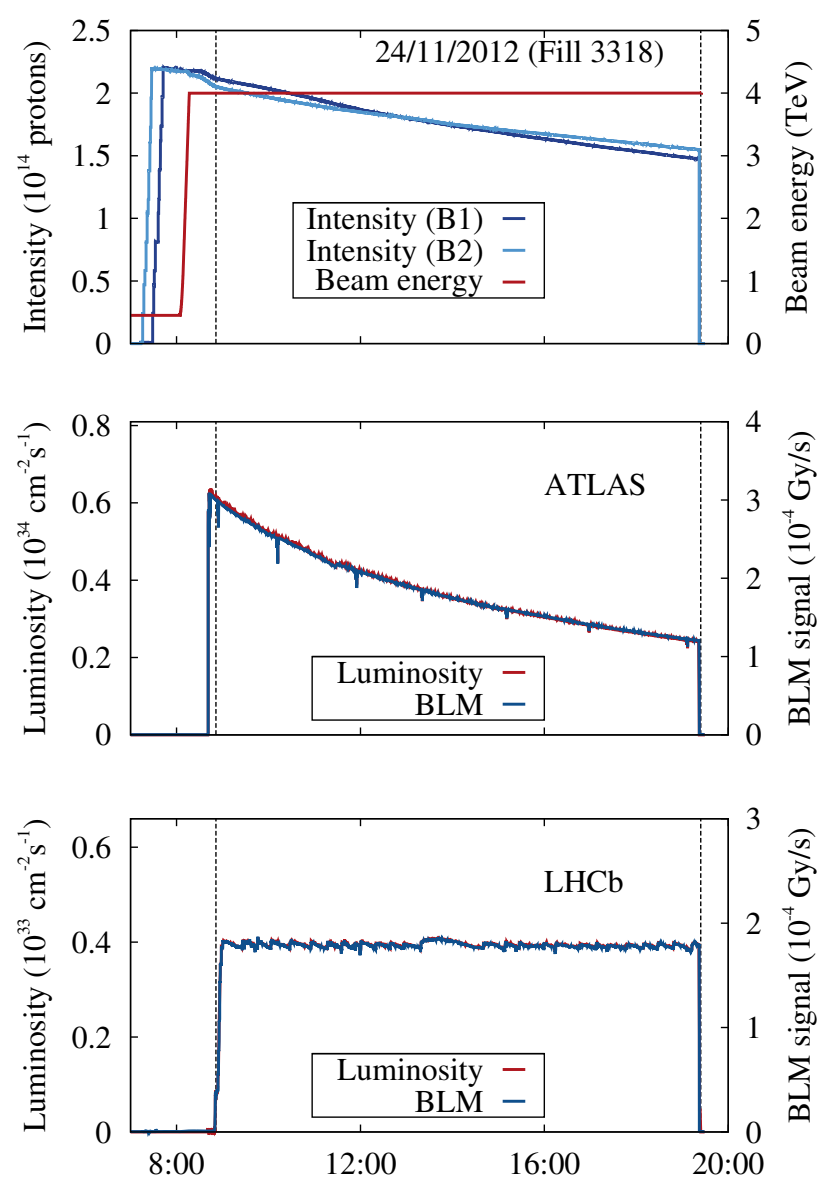

FIG. 14. Time evolution of beam intensities during a regular proton physics fill in 2012 (top) together with the instantaneous luminosity delivered to ATLAS (center) and LHCb (bottom), as well as the dose rates measured by a BLM about $23 \mathrm{~m}$ from the respective interaction point. The vertical lines indicate the time period where the LHC was in stable collision beam mode. The center-of-mass energy of the two colliding beams was $\sqrt{s}=8 \mathrm{TeV}$.

collisions (28\% escaping on each side of the experiment). Other contributions to BLM signals arise from shower particles leaking from the ATLAS forward calorimeter, vacuum chambers, and, in particular, the TAS. On the other hand, particles which are below the pseudorapidity acceptance of the forward calorimeter (these are particles with an angle larger than $\sim 5^{\circ}$ with respect to the beam axis) are not expected to significantly contribute to BLM signals, as they carry only $\sim 1.4 \%$ of the energy released in the collisions. Therefore, the absence of ATLAS detector components other than the forward calorimeter is considered justified.

The proton-proton collisions in the IPs were simulated by means of DPMJET-III. Here we use the latest version $[87,88]$, which includes several improvements based on LHC results. Collisions were generated according to the crossing schemes adopted in 2012, i.e., a vertical crossing with a half crossing angle of $145 \mu \mathrm{rad}$ in IP1 (ATLAS) and a tilted crossing plane with a half crossing angle of
$254 \mu \mathrm{rad}$ in IP8 (LHCb). The tilt in IP8 is due to vertical corrector bumps, which are superimposed upon the closed horizontal bump created by the LHCb spectrometer dipole and its compensators [89]. In this study, we consider only run periods during which the spectrometer was operated with negative polarity.

Figure 16 shows the obtained simulation results together with BLM measurements from different representative physics fills in 2012. The different fills are identified by the unique LHC fill number. All BLMs displayed in the figure are mounted on the Q1-Q3 cryostats with the exception of the most upstream BLM, which is located close to the vacuum chamber connecting the triplet with the experiment cavern. The measured signals are time integrated over the fills and are normalized to the number of inelastic proton-proton collisions given by

$$
N_{i}^{p p}=\sigma_{i}^{p p} \int \mathcal{L}(t) d t
$$

where $\sigma_{i}^{p p}$ is the inelastic cross section $(71.73 \mathrm{mb}$ at $\sqrt{s}=$ $8 \mathrm{TeV}$ [90]) and $\mathcal{L}(t)$ is the instantaneous luminosity delivered to the experiments. Each considered fill lasted at least $17 \mathrm{hr}$ and resulted in an integral luminosity $\int \mathcal{L} d t$ between 223 and $237 \mathrm{pb}^{-1}$ in ATLAS and between 21 and $27 \mathrm{pb}^{-1}$ in LHCb. To obtain the net signal from collision losses, the time-integrated dose values were corrected for the noise pedestal. For this purpose, individual BLM noise levels were derived from reference periods without a beam prior to each fill. The normalized noise-corrected signals agree within a few percent across different fills, which confirms that BLM signals in the final focus regions are dominated by luminosity production in the experiments.

As can be seen in Fig. 16, the normalized signals at the Q1 exhibit a significant difference between IR1 and IR8, which can be mainly attributed to the $1.8-\mathrm{m}$-long TAS absorber (made of copper) present only in IR1 (see Fig. 15). The simulations show that the TAS, which is located a few meters upstream of the triplet, dissipates approximately $15 \%$ of the power released per beam in ATLAS. This, in turn, reduces the power load in the first half of the Q1 as can be seen from the different BLM patterns in Fig. 16. Together with a set of additional absorbers further downstream, the TAS was designed to avoid collision-debrisinduced magnet quenches when operating at nominal energy and luminosity [22]. The TAS reduces, in addition, the dynamic heat load to be evacuated by the cryogenic system and increases the lifetime of the triplet magnets [22]. Identical absorbers are installed next to the CMS experiment in IR5, whereas no TAS is required in IR8 owing to the much lower luminosity.

The measured BLM patterns are well reproduced in the simulations, in particular, the distinct differences between IR1 and IR8 due to the absence of the TAS in the latter IR. The simulation predictions show, however, a slight tendency 


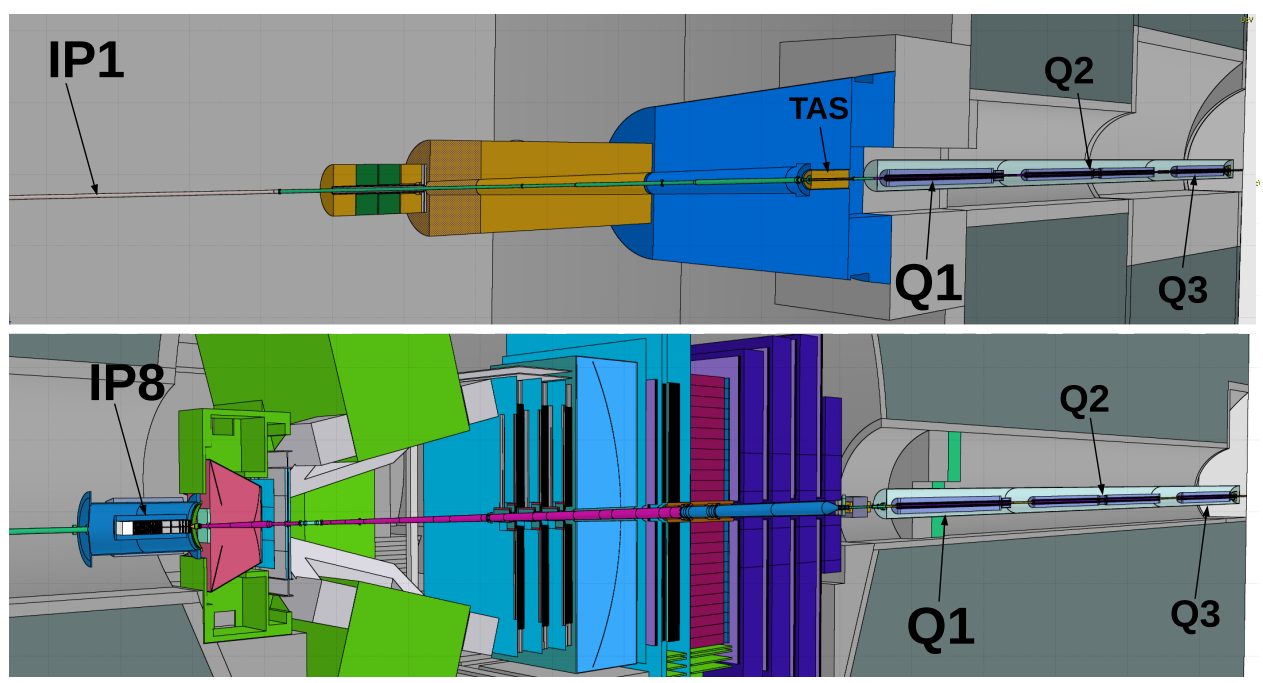

FIG. 15. Geometry models of the ATLAS (top) and LHCb (bottom) experimental regions. The first model includes only the forward calorimeter, forward shielding, and absorbers, while the latter model includes also the entire detector. The triplet quadrupoles (Q1, Q2, and Q3) can be seen on the right side. The interaction points (IPs), where the two beams collide, are located on the left.
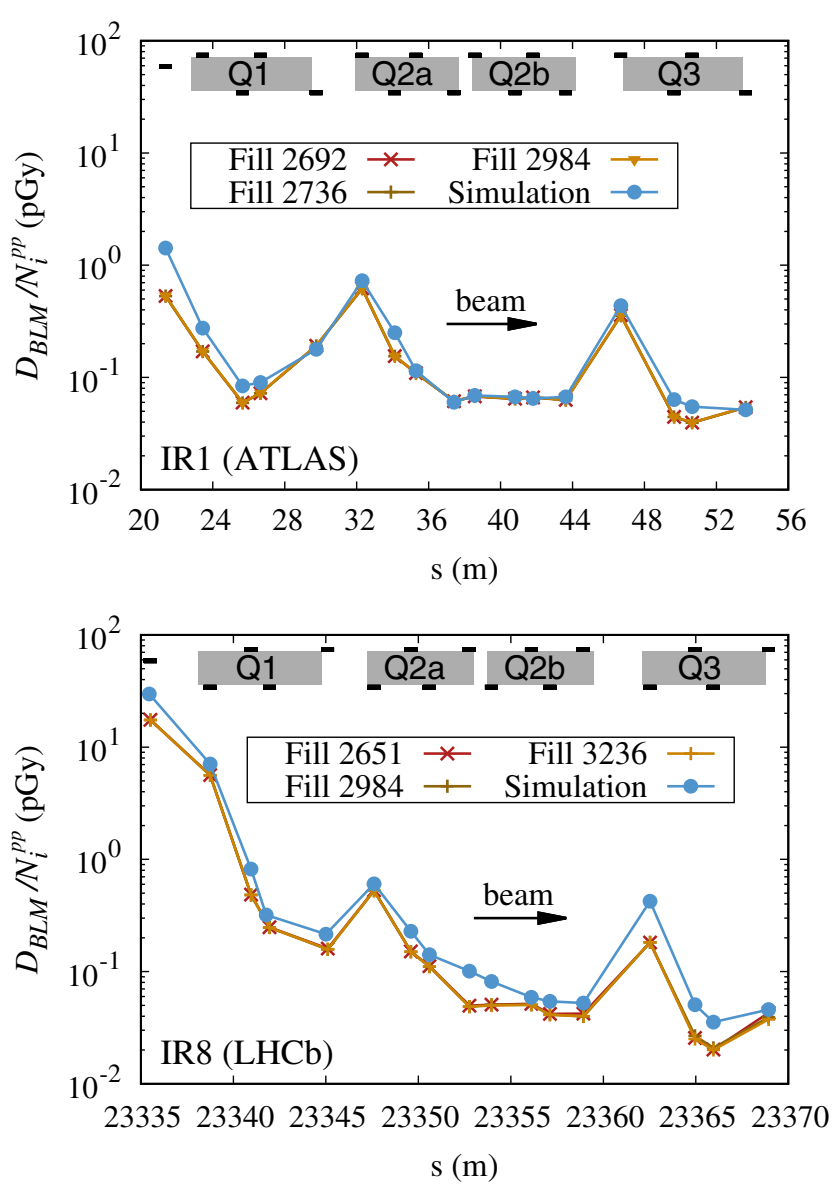

FIG. 16. Simulated and measured BLM signals along final focus quadrupoles for proton-proton collisions at a center-ofmass energy of $\sqrt{s}=8 \mathrm{TeV}$ in ATLAS (top) and LHCb (bottom). The measurements were recorded during different physics fills in 2012. All signals are per proton-proton collision in the respective interaction point. The statistical error of the simulation results is smaller than $10 \%$. to systematically overestimate measured dose values. The discrepancy is particularly visible along the Q2 and Q3 in IR8. On average, the simulated signals are about $20 \%$ higher than measured ones in IR1 and about 50\% in IR8. This discrepancy is larger than the error attributed to both the cross section measurement and the integral luminosity per fill. The error of the latter is dominated by the error of the luminosity calibrations, which is estimated to be a few percent in the case of $\mathrm{LHCb}$ [91]. A possible explanation of the discrepancies could be approximations in the geometry models. This assumption was probed in a test study, where particle transport was suppressed in some regions of IR8. The results show that BLM signals at the triplet are determined not only by showers escaping from the quadrupoles and their interconnects, but also by secondary particles generated upstream of the Q1 which travel outside of the magnets. Depending on the BLM position, these secondary particles contribute between $5 \%$ and $85 \%$ to the total BLM dose. If one would neglect this contribution, most BLM signals at the Q1 and Q2 would be lower than the measurements. The contribution of these particles shows a higher sensitivity to geometry details peripheral to the beam line, including shielding installations in the IR8 tunnel. It is possible that the contribution of these particles is overestimated. Additional sensitivity studies would be needed to assess the dependence of BLM on geometric details outside of the magnets. The overall agreement found for IR1 is comparable to the agreement observed in our previous study for CMS [36], albeit this study was for a slightly different collision energy. Although the collision plane in CMS is orthogonal to the one in ATLAS, it is not surprising that the findings are similar, since the TAS, which has a great impact on the leakage, is the same in both cases. 


\section{Bound-free pair production in heavy ion collisions}

During dedicated run periods in 2010 and 2011, the LHC collided $1.38 A \mathrm{TeV}{ }^{208} \mathrm{~Pb}^{82+}$ ion beams, achieving peak luminosities of up to $\sim 0.5 \times 10^{27} \mathrm{~cm}^{-2} \mathrm{~s}^{-1}$ in ATLAS, CMS, and ALICE. In 2015, the design luminosity of $10^{27} \mathrm{~cm}^{-2} \mathrm{~s}^{-1}$ was exceeded by a factor of 3.6 at almost double the beam energy $(2.51 \mathrm{~A} \mathrm{TeV})$ achieved in previous years [4]. In 2018, the luminosity could be further increased to more than $6 \times 10^{27} \mathrm{~cm}^{-2} \mathrm{~s}^{-1}$ in ATLAS and CMS. Certain reaction channels in ultraperipheral collisions of ${ }^{208} \mathrm{~Pb}^{82+}$ beams lead to the creation of secondary ions with a slightly changed charge-to-mass ratio, which are selectively lost at different locations in the LHC $[32,47,92,93]$. The most detrimental process for machine operation is singlephoton pair production with subsequent electron capture by one of the fully stripped ions. This process is referred to as bound-free pair production (BFPP).

The cross section for BFPP of one ion is $250 \mathrm{~b}$ at the LHC center-of-mass energy (per colliding nucleon pair) of $\sqrt{s_{N N}}=2.76 \mathrm{TeV}$ and $276 \mathrm{~b}$ at $\sqrt{s_{N N}}=5.02 \mathrm{TeV}$. The cross sections have been derived from the parameterization in Ref. [94]. The created ${ }^{208} \mathrm{~Pb}^{81+}$ ions separate from the circulating beam envelope after the long straight section and impact on the beam screen in the neighboring dispersion suppressor $[32,92,93]$. The small size of these secondary beams gives rise to very localized losses which, in turn, lead to a localized increase of BLM signals in the proximity of the impact location. BLM patterns measured during ion operation in 2010, 2011, 2015, and 2018 showed clear evidence of the presence of ${ }^{208} \mathrm{~Pb}^{81+}$ losses in the dispersion suppressors next to IR1, IR2, and IR5 [95-97].

In this section, we compare simulation predictions of BFPP-induced BLM signals against BLM measurements from the $2011{ }^{208} \mathrm{~Pb}^{82+}$ run. Preliminary results have been shown in Ref. [40]. The study focuses on BFPP ions created in IP5 (CMS) which exit the detector in the direction of the clockwise rotating beam. The BLM measurements indicate that the ${ }^{208} \mathrm{~Pb}^{81+}$ ions are lost $\sim 418-420 \mathrm{~m}$ from the IP, just upstream of the cryostat connecting the dispersion suppressor with the arc. This loss location is in good agreement with simulation predictions [32] made before the first heavy ion run in the LHC.

In this paper, we adopted the same approach as in Ref. [32] to derive the phase space distribution of ${ }^{208} \mathrm{~Pb}^{81+}$ ions impacting on the beam screen. Further details can also be found in Ref. [96]. Starting from the distribution of collision points in the IP, the envelope of the BFPP beam was tracked to the dispersion suppressor using an optical transfer matrix. As a second step, individual particle trajectories were sampled in the dispersion suppressor, and their intersection with the aperture was calculated. The obtained impact distribution has a full width at half maximum of about $1.5 \mathrm{~m}$ in the beam direction [96]. The average impact angle of ions on the beam screen is about $420 \mu \mathrm{rad}$ [96].
Based on the loss distribution, the BLM response was calculated with FLUKA for a series of eight BLMs around the impact location. To decrease the computational time, the secondary particle multiplicity in nuclear interactions was biased by a factor of 0.2 . The geometry model used in the simulations was similar to the one shown in Fig. 1, with the addition of the connection cryostat.

Figure 17 compares the obtained results with BLM signals measured during different representative physics fills. The peak of the assumed loss distribution occurs around the interconnect between the connection cryostat (LEGR) and a main bending dipole (MB). As in the previous section, measured signals were time integrated over the fills and were normalized to the number of singlephoton bound-free pair productions given by

$$
N_{B F P P}=\sigma_{B F P P} \int \mathcal{L}(t) d t
$$

where $\sigma_{B F P P}$ is the bound-free pair production cross section and $\mathcal{L}(t)$ is the instantaneous luminosity measured by the experiment. The integrated luminosity per fill ranged from 4.2 to $6.2 \mu \mathrm{b}^{-1}$, which corresponds to about $1-1.5 \times 10^{9}$ bound-free pair productions per beam and fill. As in the previous section, the noise pedestal was subtracted from the measured BLM signals. For all BLMs, the signal-to-noise ratio was larger than 2 , even at the end of fills when the instantaneous luminosity had decreased to about 30\%-40\% of its initial value because of burnoff. The normalized BLM signals from the different fills agree within $4 \%$ for the BLM with the highest dose value. For the other BLMs, the measured signals agree within $10 \%$, except for the two

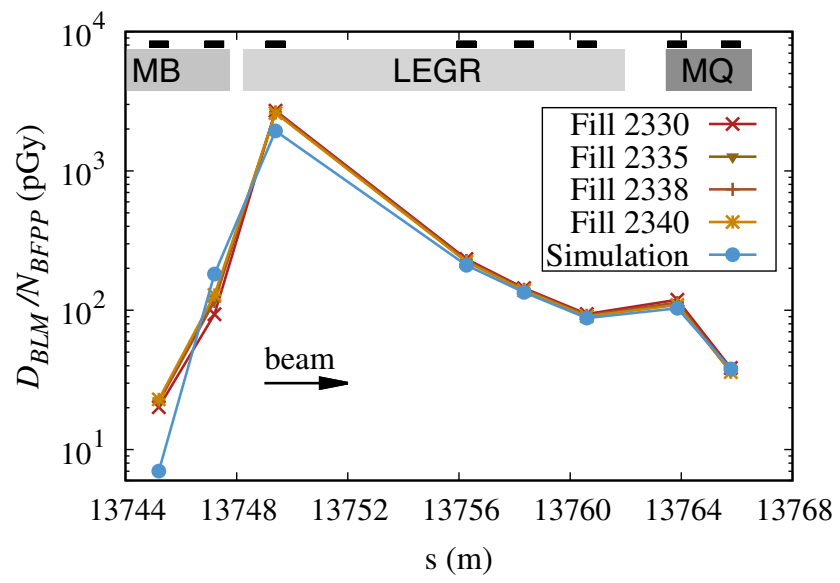

FIG. 17. Simulated and measured BLM signals for $1.38 A \mathrm{TeV}$ ${ }^{208} \mathrm{~Pb}^{81+}$ ion losses in the dispersion suppressor next to IR5. The measurements were recorded during different fills in 2011. Signals are normalized to the number of bound-free pair productions per beam in IP5. The beam direction is from the left to the right. Statistical errors of simulation results are less than $10 \%$ for BLMs downstream of the assumed loss location $(s \approx 13748 \mathrm{~m}$ ), while they are up to $20 \%$ for upstream BLMs. 
upstream monitors, where differences of up to $30 \%$ are found. This fill-to-fill variation highlights the uncertainty of the measurements, which is larger for signals closer to the noise level.

The simulation results agree with measured signals within 30\% for all BLMs downstream of the peak location of losses. Larger discrepancies of up to a factor of 2.5 are observed for the two upstream monitors, which is more than the above-mentioned fill-to-fill variation. These discrepancies could be due to an offset between the assumed and the actual loss location, for example, due to a nonideal beam screen or vacuum chamber alignment. During the ${ }^{208} \mathrm{~Pb}^{82+}$ runs, an asymmetry of BFPP-induced BLM patterns was observed between the left and right sides of IP5, indicating that the left and right apertures might not be fully identical. This can be explained by the mechanical aperture tolerance of LHC arc dipoles, which is about $1 \mathrm{~mm}$. Considering the grazing impact angle of BFPP ions on the beam screen, it is not unreasonable that the actual impact location can deviate from the nominal one.

\section{CONCLUSIONS}

This paper demonstrated the predictive power of particle shower simulations for quantifying the beam-induced energy deposition in the LHC. The benchmark studies were based on ionization chamber measurements acquired during 3.5 and $4 \mathrm{TeV}$ proton operation and $1.38 \mathrm{ATeV}$ ${ }^{802} \mathrm{~Pb}^{82+}$ ion operation in LHC run I.

The beam loss test with the wire scanner provided almost ideal conditions for benchmarking energy deposition simulations for pointlike beam losses on an obstacle. Transient beam losses on obstacles were the main cause of beaminduced quenches and BLM aborts in LHC run II $[15,16,19]$, including such cases as macroparticles entering into the beams and beam losses on the macroscopic object in a bending dipole, which was observed for the first time in 2015 when restarting the LHC after a 2-yr shutdown. The wire scanner benchmark shows that energy deposition measurements for such pointlike source terms can be reproduced with good accuracy albeit differences for individual BLMs can amount to a few tens of percent. This demonstrates that essential information can be reconstructed for such beam losses, like the number of inelastic nuclear collisions of beam particles inside the object. This information can be used to assess, for example, the showerinduced energy deposition density in magnets, which is important for validating quench margins and adjusting beam abort thresholds.

The benchmarks against dust-particle-induced BLM signals illustrate that shower simulations can also be used to narrow down the source location of losses. This is possible because of the strong dependence of spatial BLM patterns on the loss point. The analysis of different dust events demonstrate that, by adjusting the loss location in the simulation, a good match between measured and simulated patterns can be found. It is estimated that the position of the collision vertex can be reconstructed with an accuracy of about one meter. This method has been applied in LHC run II to locate the obstacle in the aforementioned dipole and to narrow down the source location of recurring beam loss events in 2017 [19].

Luminosity-driven beam losses are a significant source of power loss in a collider like the LHC. The BLM benchmarks for inelastic proton-proton collisions and bound-free pair production in ${ }^{208} \mathrm{~Pb}^{82+}$ ion collisions show a good agreement between simulations and measurements, although a systematic overestimation of about $50 \%$ was found in the case of proton-proton collisions in LHCb and to a smaller extent also in the case of ATLAS. This discrepancy can possibly be attributed to approximations in the geometry model, which lead to an overestimation of the shower leakage to BLMs.

A good agreement is also found for more complex source terms like collimation losses, where a sophisticated setup of tracking and particle interaction simulations is needed to account for multiturn effects. Measured and simulated BLM signals in the betatron collimation insertion, which are governed by showers escaping from collimators, agree within a few tens of percent for a good fraction of the BLMs. Larger discrepancies are found for the adjacent dispersion suppressor, where BLM signals are dominated by single diffractive protons escaping from the cleaning system. Simulation predictions are a factor of 3 lower than the measurements, which can likely be attributed to an underestimation of escaping protons. The most probable cause for this underestimation is the presence of imperfections which are not accounted for in the simulations. These imperfections include collimator tilts, collimator gap errors, a nonideal centering of collimators around the beam (e.g., because of orbit shifts), or aperture misalignments. Previous tracking studies showed that a tilt of primary collimator jaws can increase the proton leakage by about a factor of 2. Energy deposition studies, which take into account imperfections, are planned to be presented in a future publication.

This paper demonstrates that Monte Carlo shower simulations are a powerful method for analyzing beam losses and their consequences in high-energy proton and ion colliders. Having a good understanding of the showerinduced energy deposition in machine components is essential for defining operational parameters, for estimating the lifetime of equipment, and for designing new hardware for future operation at higher intensities and luminosities. The simulation models and methods presented in this paper are employed in various studies $[23,25,38,39,42]$ for the High Luminosity (HL) upgrade of the LHC [98]. These studies are the basis for the conceptual design of HL-LHC protection systems, which are needed to avoid quenches and long-term radiation damage and to protect the machine in case of beam loss accidents. Similar simulation methods 
are also adopted for the design of future high-energy machines, for example, within the Future Circular Collider study $[99,100]$. The results derived in this paper provide an important assessment of the predictive ability of shower studies for such accelerator environments.

\section{ACKNOWLEDGMENTS}

We are grateful to A. Mariet and R. Veness from the CERN Beam Instrumentation group for performing density measurements of the carbon wires used in the LHC wire scanner. We thank S. Roesler and C. Theis from the CERN Radiation Protection group, as well as L. Shektman and M. Karacson from the LHCb Collaboration for the FLUKA model of the LHCb detector, shielding, and cavern. We are grateful to A. Lambert for English proofreading of this manuscript.

[1] O. S. Brüning, P. Collier, P. Lebrun, S. Myers, R. Ostojic, J. Poole, and P. Proudlock, LHC design report, CERN Yellow Reports: Monographs (CERN, Geneva, 2004).

[2] M. Lamont, The first years of LHC operation for luminosity production, in Proceedings of the 4th International Particle Accelerator Conference, IPAC-2013, Shanghai, China, 2013 (JACoW, Geneva, Switzerland, 2013), MOYAB101, pp. 6-10.

[3] J.M. Jowett, Colliding heavy ions in the LHC, in Proceedings of the 9th International Particle Accelerator Conference, Vancouver, Canada, 2018 (JACoW, Geneva, Switzerland, 2018), TUXGBD2, pp. 584-589, http://dx .doi.org/10.18429/JACoW-IPAC2018-TUXGBD2.

[4] J. M. Jowett et al., The 2015 heavy-ion run of the LHC, in Proceedings of the 7th International Particle Accelerator Conference, Busan, Korea (JACoW, Geneva, Switzerland, 2016), TUPMW027, pp. 1493-1496, http://dx.doi .org/10.18429/JACoW-IPAC2016-TUPMW027.

[5] R. Steerenberg and J. Wenninger, Operational challenges and performance for the LHC during run II, in Proceedings of the 61st ICFA Advanced Beam Dynamics Workshop on High-Intensity and High-Brightness Hadron Beams, Daejeon, Korea (JACoW, Geneva, Switzerland, 2018), THA2WD01, pp. 357-361, https://doi.org/10.18429/ JACoW-HB2018-THA2WD01.

[6] R. Assmann, Collimators and beam absorbers for cleaning and machine protection, in Proceedings of the 2nd LHC Project Workshop - Chamonix XIV, CERN, Geneva, Switzerland, edited by J. Poole (CERN, Geneva, Switzerland, 2005), pp. 261-267.

[7] R. W. Assmann et al., The final collimation system for the LHC, in Proceedings of the 10th European Particle Accelerator Conference, Edinburgh, Scotland, 2006 (EPS-AG, Edinburgh, Scotland, 2006), TUODFI01, pp. 986-988.

[8] T. Baer et al., UFOs in the LHC, in Proceedings of the 2nd International Particle Accelerator Conference, San Sebastián, Spain (EPS-AG, Spain, 2011), TUPC137, pp. 1347-1349.
[9] E. Nebot et al., Analysis of fast losses in the LHC with the BLM system, in Proceedings of the 2nd International Particle Accelerator Conference, San Sebastián, Spain (EPS-AG, Spain, 2011), TUPC136, pp. 1344-1346.

[10] T. Baer et al., UFOs in the LHC: Observations, studies, and extrapolations, in Proceedings of the 3rd International Particle Accelerator Conference, New Orleans, LA, 2012 (IEEE, Piscataway, NJ, 2012), THPPP086, pp. 39363938.

[11] B. Goddard et al., Transient beam losses in the LHC injection kickers from micron scale dust particles, in Proceedings of the 3rd International Particle Accelerator Conference, New Orleans, LA, 2012 (IEEE, Piscataway, NJ, 2012), TUPPR092, pp. 2044-2046.

[12] T. Baer, Very fast losses of the circulating LHC beam, their mitigation and machine protection, Ph.D. thesis, CERN and Hamburg University, 2013.

[13] B. Auchmann, J. Ghini, L. Grob, G. Iadarola, A. Lechner, and G. Papotti, How to survive an UFO attack?, in Proceedings of the 6th Evian Workshop on LHC Beam Operation, Evian Les Bains, France (CERN, Geneva, Switzerland, 2015), pp. 81-86.

[14] G. Papotti, M. Albert, B. Auchmann, E. B. Holzer, M. Kalliokoski, and A. Lechner, Macroparticle-induced losses during 6.5 TeV LHC operation, in Proceedings of the 7th International Particle Accelerator Conference, Busan, South Korea (JACoW, Geneva, Switzerland, 2016), TUPMW023, pp. 1481-1484, http://dx.doi.org/10.18429/ JACoW-IPAC2016-TUPMW023.

[15] A. Lechner et al., BLM thresholds and UFOs, in Proceedings of the 7th Evian Workshop on LHC Beam Operation, Evian Les Bains, France (CERN, Geneva, Switzerland, 2016), pp. 209-214.

[16] D. Mirarchi, R. Bruce, M. Giovannozzi, P. Hermes, S. Redaelli, B. Salvachua, G. Valentino, and J. Wenninger, LHC aperture and ULO restrictions: Are they a possible limitation in 2016?, in Proceedings of the 6th Evian Workshop on LHC Beam Operation, Evian Les Bains, France (CERN, Geneva, Switzerland, 2016), pp. 87-94.

[17] J. Jiménez et al., Observations, analysis and mitigation of recurrent LHC beam dumps caused by fast losses in arc half-cell 16L2, in Proceedings of the 9th International Particle Accelerator Conference, Vancouver, Canada (JACoW, Geneva, Switzerland, 2018), MOPMF053, pp. 228-231, http://dx.doi.org/10.18429/JACoW-IPAC 2018-MOPMF053.

[18] B. Salvant et al., Experimental characterisation of a fast instability linked to losses in the $16 \mathrm{~L} 2$ cryogenic half-cell in the CERN LHC, in Proceedings of the 9th International Particle Accelerator Conference, Vancouver, Canada (JACoW, Geneva, Switzerland, 2018), THPAF058, pp. 3103-3106, http://dx.doi.org/10 .18429/JACoW-IPAC2018-THPAF058.

[19] A. Lechner et al., Beam loss measurements for recurring fast loss events during 2017 operation possibly caused by macroparticles, in Proceedings of the 9th International Particle Accelerator Conference, Vancouver, Canada (JACoW, Geneva, Switzerland, 2018), TUPAF040, pp. 780-784, http://dx.doi.org/10.18429/JACoW-IPAC 2018-TUPAF040. 
[20] A. Lechner, A. Alnuaimi, C. Bracco, F. Cerutti, A. Christov, L. S. Esposito, N. V. Shetty, and V. Vlachoudis, Energy deposition studies for fast losses during LHC injection failures, in Proceedings of the 4th International Particle Accelerator Conference, Shanghai, China, 2013 (JACoW, Geneva, Switzerland, 2013), TUPFI027, pp. 1397-1399.

[21] B. Auchmann et al., Testing beam-induced quench levels of LHC superconducting magnets, Phys. Rev. Accel. Beams 18, 061002 (2015).

[22] N. Mokhov, I. Rakhno, J. Kerby, and J. Strait, Protecting LHC IP1/IP5 components against radiation resulting from colliding beam interactions, Technical Report No. LHCProject-Report-633, CERN, 2003.

[23] N. V. Mokhov, I. L. Rakhno, I. S. Tropin, F. Cerutti, L. S. Esposito, and A. Lechner, Energy deposition studies for the high-luminosity Large Hadron Collider inner triplet magnets, Phys. Rev. Accel. Beams 18, 051001 (2015).

[24] N. V. Mokhov, Beam-Materials Interactions, Rev. Accel. Sci. Techol. 06, 275 (2013).

[25] C. Bahamonde Castro, F. Cerutti, P. Fessia, A. Lechner, A. Mereghetti, D. Mirarchi, S. Redaelli, and E. Skordis, Improved protection of the warm magnets of the LHC betatron cleaning insertion, in Proceedings of the 8th International Particle Accelerator Conference, Copenhagen, Denmark (JACoW, Geneva, Switzerland, 2017), MOPAB004, pp. 72-75, http://dx.doi.org/10 .18429/JACoW-IPAC2017-MOPAB004.

[26] H. Vincke, C. Theis, and S. Roesler, Induced radioactivity in and around high-energy particle accelerators, Radiation Protection Dosimetry 146, 434 (2011).

[27] R. Bruce et al., Sources of machine-induced background in the ATLAS and CMS detectors at the CERN Large Hadron Collider, Nucl. Instrum. Methods Phys. Res., Sect. A 729, 825 (2013).

[28] A. L. Perrot et al., Optimisation and implementation of the R2E shielding and relocation mitigation measures at the LHC during the LS1, in Proceedings of the 5th International Particle Accelerator Conference, Dresden, Germany (JACoW, Geneva, Switzerland, 2014), TUPRO012, pp. 1027-1029, https://doi.org/10.18429/ JACoW-IPAC2014-TUPRO012.

[29] A. Ferrari, P. Sala, A. Fassò, and J. Ranft, FLUKA: A multi-particle transport code, Technical Report No. CERN-2005-10, INFN/TC_05/11, SLAC-R-773, CERN/INFN, 2005.

[30] T. Böhlen, F. Cerutti, M. Chin, A. Fassò, A. Ferrari, P. G. Ortega, A. Mairani, P. R. Sala, G. Smirnov, and V. Vlachoudis, The FLUKA code: Developments and challenges for high energy and medical applications, Nucl. Data Sheets 120, 211 (2014).

[31] M. Sapinski, F. Cerutti, B. Dehning, A. Ferrari, C. Hoa, A. Lechner, M. Mauri, and A. Mereghetti, Estimation of thresholds for the signals of the BLMs around the LHC final focus triplet magnets, in Proceedings of the 3rd International Particle Accelerator Conference, New Orleans, LA, 2012 (IEEE, Piscataway, NJ, 2012), THPPR037, pp. 4053-4055.

[32] R. Bruce, D. Bocian, S. Gilardoni, and J. M. Jowett, Beam losses from ultraperipheral nuclear collisions between
${ }^{208} \mathrm{~Pb}^{82+}$ ions in the Large Hadron Collider and their alleviation, Phys. Rev. Accel. Beams 12, 071002 (2009).

[33] F. Cerutti, A. Ferrari, A. Mereghetti, E. Todesco, and E. Wildner, Ruling factors in the impact of collision debris on the LHC high luminosity insertion magnets, in Proceedings of the International Topical Meeting on Nuclear Research Applications and Utilization of Accelerators, Vienna, Austria (IAEA, Vienna, Austria, 2009), pp. 1-8.

[34] G. Battistoni et al., FLUKA capabilities and CERN applications for the study of radiation damage to electronics at high-energy hadron accelerators, Prog. Nucl. Sci. Tech. 2, 948 (2011).

[35] K. Roed, V. Boccone, M. Brugger, A. Ferrari, D. Kramer, E. Lebbos, R. Losito, A. Mereghetti, G. Spiezia, and R. Versaci, FLUKA simulations for SEE studies of critical LHC underground areas, IEEE Trans. Nucl. Sci. 58, 932 (2011).

[36] V. Boccone et al., Beam-machine interaction at the CERN LHC, Nucl. Data Sheets 120, 215 (2014).

[37] R. Bruce et al., Simulations and measurements of beam loss patterns at the CERN Large Hadron Collider, Phys. Rev. Accel. Beams 17, 081004 (2014).

[38] A. Lechner et al., Power deposition in LHC magnets with and without dispersion suppressor collimators downstream of the betatron cleaning insertion, in Proceedings of the 5th International Particle Accelerator Conference, Dresden, Germany (JACoW, Geneva, Switzerland, 2014), MOPRO021, pp. 112-115, http://dx.doi.org/10.18429/ JACoW-IPAC2014-MOPRO021.

[39] A. Lechner et al., Protection of superconducting magnets in case of accidental beam losses during HL-LHC injection, in Proceedings of the 6th International Particle Accelerator Conference, Richmond, Virginia, USA (JACoW, Geneva, Switzerland, 2015), TUPTY049, pp. 2128-2131, https://doi.org/10.18429/JACoW-IPAC 2015-TUPTY049.

[40] N. Shetty, F. Cerutti, A. Ferrari, A. Lechner, E. Skordis, and V. Vlachoudis, Nuclear reactions in the context of LHC operation, in Proceedings of the 14th International Conference on Nuclear Reaction Mechanisms, Villa Monastero, Varenna, Italy (CERN, Geneva, Switzerland, 2015), pp. 313-318.

[41] E. Skordis, R. Bruce, F. Cerutti, A. Ferrari, P. Hermes, A. Lechner, A. Mereghetti, P. Ortega, S. Redaelli, and V. Vlachoudis, Impact of beam losses in the LHC collimation regions, in Proceedings of the 6th International Particle Accelerator Conference, Richmond, Virginia, USA(JACoW, Geneva, Switzerland, 2015), TUPTY046, pp. 2116-2119, https://doi.org/10.18429/JACoW-IPAC2015-TUPTY046.

[42] C. Bahamonde Castro et al., Power deposition in LHC magnets due to bound-free pair production in the experimental insertions, in Proceedings of the 7th International Particle Accelerator Conference, Busan, South Korea (JACoW, Geneva, Switzerland, 2016), TUPMW006, pp. 1418-1421, http://dx.doi.org/10.18429/JACoWIPAC2016-TUPMW006.

[43] E. Skordis et al., Study of the 2015 top energy LHC collimation quench tests through an advanced simulation chain, in Proceedings of the 8th International Particle 
Accelerator Conference, Copenhagen, Denmark (JACoW, Geneva, Switzerland, 2017), MOPAB012, pp. 100-103, http://dx.doi.org/10.18429/JACoW-IPAC 2017-МОРАВ012.

[44] E. Quaranta, A. Bertarelli, R. Bruce, F. Carra, F. Cerutti, A. Lechner, S. Redaelli, E. Skordis, and P. Gradassi, Modeling of beam-induced damage of the LHC tertiary collimators, Phys. Rev. Accel. Beams 20, 091002 (2017).

[45] M. Frankl, W. Bartmann, M. Bednarek, C. Bracco, A. Lechner, A. Verweij, C. Wiesner, and D. Wollmann, Energy deposition studies and analysis of the quench behavior in the case of asynchronous dumps during 6.5 TeV LHC proton beam operation, in Proceedings of the 9th International Particle Accelerator Conference, Vancouver, Canada (JACoW, Geneva, Switzerland, 2018), TUPAF028, pp. 736-739, http://dx.doi.org/10 .18429/JACoW-IPAC2018-TUPAF028.

[46] G. Battistoni, F. Cerutti, A. Fassó, A. Ferrari, S. Muraro, J. Ranft, S. Roesler, and P. R. Sala, The FLUKA code: Description and benchmarking, AIP Conf. Proc. 896, 31 (2007).

[47] H. H. Braun, A. Fassò, A. Ferrari, J. M. Jowett, P. R. Sala, and G. I. Smirnov, Hadronic and electromagnetic fragmentation of ultrarelativistic heavy ions at LHC, Phys. Rev. Accel. Beams 17, 021006 (2014).

[48] E. B. Holzer et al., Beam loss monitoring system for the LHC, in Proceedings of the 2005 IEEE Nuclear Science Symposium Conference Record, San Juan/Puerto Rico (IEEE, Piscataway, NJ, 2005), pp. 1052-1056, https://doi .org/10.1109/NSSMIC.2005.1596433.

[49] B. Dehning et al., The LHC beam loss measurement system, in Proceedings of the 22nd Particle Accelerator Conference, PAC-2007, Albuquerque, NM (IEEE, Piscataway, NJ, 2007), FRPMN071, pp. 4192-4194, https://doi.org/10.1109/PAC.2007.4439980.

[50] M. Stockner, B. Dehning, C. Fabjan, G. Ferioli, and E. B. Holzer, Measurements and simulations of ionization chamber signals in mixed radiation fields for the LHC BLM system, in Proceedings of the 2006 IEEE Nuclear Science Symposium Conference Record (IEEE, Piscataway, NJ, 2006), Vol. 3, pp. 1342-1345, https://doi .org/10.1109/NSSMIC.2006.354151.

[51] M. Stockner, Beam loss calibration studies for high energy proton accelerators, Ph.D. thesis, CERN and Vienna University of Technology, 2007.

[52] M. Stockner, B. Dehning, C. Fabjan, E. B. Holzer, and D. Kramer, Classification of the LHC BLM ionization chamber, in Proceedings of the 8th European Workshop on Beam Diagnostics and Instrumentation for Particle Accelerators, Venice, Italy (2007), WEPC09, pp. 328-330.

[53] L. Sarchiapone, M. Brugger, B. Dehning, D. Kramer, M. Stockner, and V. Vlachoudis, FLUKA Monte Carlo simulations and benchmark measurements for the LHC beam loss monitors, Nucl. Instrum. Methods Phys. Res., Sect. A 581, 511 (2007).

[54] E. Lebbos et al., Measurements and simulations of the BLM response to a radiation field inside the CERF target area, Technical Report No. CERN-EN-Note-2010-001, CERN, 2010.
[55] M. Sapinski et al., Beam induced quench tests of LHC magnets, in Proceedings of the 4th International Particle Accelerator Conference, IPAC-2013, Shanghai, China, 2013 (JACoW, Geneva, Switzerland, 2013), THPEA045, pp. 3243-3245.

[56] M. Sapinski et al., Beam-induced quench tests of LHC magnets, in Proceedings of the 5th International Particle Accelerator Conference, Dresden, Germany (JACoW, Geneva, Switzerland, 2014), MOOCB01, pp. 52-55, https://doi.org/10.18429/JACoW-IPAC2014-MOOCB01.

[57] M. Sapinski et al., LHC magnet quench test with beam loss generated by wire scan, in Proceedings of the 2nd International Particle Accelerator Conference, San Sebastián, Spain (EPS-AG, Spain, 2011), WEPC173, pp. 2391-2393.

[58] E. Skordis, V. Vlachoudis, R. Bruce, F. Cerutti, A. Ferrari, A. Lechner, A. Mereghetti, P. Ortega, S. Redaelli, and D. S. Pastor, FLUKA coupling to Sixtrack, in Proceedings of the ICFA Mini-Workshop on Tracking for Collimation, Geneva, Switzerland, 2015, CERN Yellow Reports: Conference Proceedings, CERN-2018-011-CP, Vol. 2, edited by S. Redaelli (CERN, Geneva, Switzerland, 2015), pp. 17-25, http://dx.doi.org/10.23732/CYRCP2018-002.

[59] S. Roesler, R. Engel, and J. Ranft, The Monte Carlo event generator DPMJET-III, in Advanced Monte Carlo for Radiation Physics, Particle Transport Simulation and Applications, edited by A. Kling, F. J.C. Baräo, M. Nakagawa, L. Távora, and P. Vaz (Springer, Berlin, 2001), pp. 1033-1038.

[60] A. Mereghetti, V. Boccone, F. Cerutti, R. Versaci, and V. Vlachoudis, The Fluka Linebuilder and element database: Tools for building complex models of accelerators beam lines, in Proceedings of the 3rd International Particle Accelerator Conference, New Orleans, LA, 2012 (IEEE, Piscataway, NJ, 2012), WEPPD071, pp. 2687-2689.

[61] V. Vlachoudis, FLAIR: A powerful but user friendly graphical interface for FLUKA, in Proceedings of the International Conference on Mathematics, Computational Methods and Reactor Physics (M\&C 2009), Saratoga Springs, New York (American Nuclear Society, Inc., Illinois, USA, 2009).

[62] S. Russenschuck, ROXIE: A computer code for the integrated design of accelerator magnets, in Proceedings of the 6th European Particle Accelerator Conference, Stockholm (IOP, London, 1998), pp. 2017-2019.

[63] http://cern.ch/madx.

[64] H. Grote and F. Schmidt, MAD-X-An upgrade from MAD8, in Proceedings of the 2003 Particle Accelerator Conference, Portland, OR (IEEE, Piscataway, NJ, 2003), FPAG014, pp. 3497-3499, https://doi.org/10.1109/PAC .2003 .1289960 .

[65] L. Deniau et al., Upgrade of MAD-X for HL-LHC project and FCC studies, in Proceedings of the 13th International Computational Accelerator Physics Conference, Key West, FL (JACoW, Geneva, Switzerland, 2018), TUPAF01, pp. 1-7, https://doi.org/10.18429/JACoW-ICAP2018 -TUPAF01.

[66] F. Schmidt, SIXTRACK, users reference manual, Technical Report No. SL/94-56 (AP), CERN, 1994. 
[67] G. Robert-Demolaize, R. Assmann, S. Redaelli, and F. Schmidt, A new version of SIXTRACK with collimation and aperture interface, in Proceedings of the 21st Particle Accelerator Conference, Knoxville, TN, 2005 (IEEE, Piscataway, NJ, 2005), FPAT081, pp. 4084-4086, https://doi.org/10.1109/PAC.2005.1591725.

[68] R. De Maria et al., SixTrack project: Status, runtime environment, and new developments, in Proceedings of the 13th International Computational Accelerator Physics Conference, Key West, FL (JACoW, Geneva, Switzerland, 2018), TUPAF02, pp. 1-7, https://doi.org/10.18429/ JACoW-ICAP2018-TUPAF02.

[69] B. Salvachua et al., Collimation quench test with $4 \mathrm{TeV}$ proton beams, Technical Report No. CERN-ACC-NOTE2014-0036, CERN, 2014.

[70] A. Mariet and R. Veness (personal communication).

[71] M. Sapinski, B. Dehning, A. Guerrero, M. Meyer, and T. Kroyer, Carbon fiber damage in particle beam, in Proceedings of the 46th ICFA Advanced Beam Dynamics Workshop on High-Intensity and High-Brightness Hadron Beams, Morschach, Switzerland, 2010 (2011), MOPD61, pp. 231-234.

[72] R. W. Assmann et al., Collimator losses in the DS of IR7 and quench test at $3.5 \mathrm{TeV}$, Technical Report No. CERNATS-Note-2011-042 MD, CERN, 2011.

[73] B. Salvachua et al., Collimation quench test with $6.5 \mathrm{TeV}$ proton beams, Technical Report No. CERN-ACC-NOTE2016-0015, CERN, 2016.

[74] A. Mereghetti et al., Sixtrack-FLUKA active coupling for the upgrade of the SPS scrapers, in Proceedings of the 4th International Particle Accelerator Conference, IPAC2013, Shanghai, China, 2013 (JACoW, Geneva, Switzerland, 2013), WEPEA064, pp. 2657-2659.

[75] H. Saeki, T. Momose, and H. Ishimaru, Observations of dust trapping phenomena in the TRISTAN accumulation ring and a study of dust removal in a beam chamber, Rev. Sci. Instrum. 62, 874 (1991).

[76] D. Sagan, Mass and charge measurement of trapped dust in the CESR storage ring, Nucl. Instrum. Methods Phys. Res., Sect. A 330, 371 (1993).

[77] F. Zimmermann, J. T. Seeman, M. Zolotorev, and W. Stoeffl, Trapped macroparticles in electron storage rings, in Proceedings of the 16th Particle Accelerator Conference, Dallas, Texas, 1995 (IEEE, Piscataway, NJ, 1995), WPG10, pp. 517-519, https://doi.org/10 .1109/PAC.1995.504705.

[78] D. R. Kelly, Dust in accelerator vacuum systems, in Proceedings of the Particle Accelerator Conference, Vancouver, BC, Canada, 1997 (IEEE, Piscataway, NJ, 1997), pp. 3547-3550, https://doi.org/10.1109/PAC.1997 .753270 .

[79] A. Kling, Dust macroparticles in HERA and DORIS, in Proceedings of the 10th European Particle Accelerator Conference, Edinburgh, Scotland, 2006 (EPS-AG, Edinburgh, Scotland, 2006), TUPLS002, pp. 1486-1488.

[80] Y. Tanimoto, T. Honda, and S. Sakanaka, Experimental demonstration and visual observation of dust trapping in an electron storage ring, Phys. Rev. Accel. Beams 12, 110702 (2009).
[81] Y. Suetsugu, K. Shibata, T. Ishibashi, K. Kanazawa, M. Shirai, S. Terui, and H. Hisamatsu, First commissioning of the SuperKEKB vacuum system, Phys. Rev. Accel. Beams 19, 121001 (2016).

[82] F. Zimmermann, M. Giovannozzi, and A. Xagkoni, Interaction of macro-particles with LHC proton beam, in Proceedings of the 1st International Particle Accelerator Conference, Kyoto, Japan (Asian Committee for Future Accelerators, 2010), MOPEC016, pp. 492-494.

[83] N. Fuster Martinez, F. Zimmermann, T. Baer, M. Giovannozzi, E. B. Holzer, E. Nebot, A. Nordt, M. Sapinski, and Z. Yang, Simulation studies of macroparticles falling into the LHC proton beam, in Proceedings of the 2nd International Particle Accelerator Conference, San Sebastian, Spain (EPS-AG, Spain, 2011), MOPS017, pp. 634-636.

[84] S. Rowan, A. Apollonio, B. Auchmann, A. Lechner, O. Picha, W. Riegler, H. Schindler, R. Schmidt, and F. Zimmermann, Interactions between macroparticles and high-energy proton beams, in Proceedings of the 6th International Particle Accelerator Conference, Richmond, VA (JACoW, Geneva, Switzerland, 2015), TUPTY045, pp. 2112-2115, https://doi.org/10.18429/ JACoW-IPAC2015-TUPTY045.

[85] R. Bruce, C. Bracco, R. D. Maria, M. Giovannozzi, A. Mereghetti, D. Mirarchi, S. Redaelli, E. Quaranta, and B. Salvachua, Reaching record-low $\beta^{*}$ at the CERN Large Hadron Collider using a novel scheme of collimator settings and optics, Nucl. Instrum. Methods Phys. Res., Sect. A 848, 19 (2017).

[86] R. Alemany-Fernandez, F. Follin, and R. Jacobsson, The LHCb online luminosity control and monitoring, in Proceedings of the 4th International Particle Accelerator Conference, IPAC-2013, Shanghai, China, 2013 (JACoW, Geneva, Switzerland, 2013), TUPFI010, pp. 1346-1348.

[87] A. Fedynitch, Cascade equations and hadronic interactions at very high energies, Ph.D. thesis, CERN and Karlsruhe Institute of Technology, 2015.

[88] A. Fedynitch and R. Engel, Revision of the high energy hadronic interaction models PHOJET/DPMJET-III, in Proceedings of the 14th International Conference on Nuclear Reaction Mechanisms, Villa Monastero, Varenna, Italy (CERN, Geneva, Switzerland, 2015), pp. 291-299.

[89] R. Alemany-Fernandez, F. Follin, B. J. Holzer, D. Jacquet, R. Versteegen, and J. Wenninger, Study and operational implementation of a tilted crossing angle in $\mathrm{LHCb}$, in Proceedings of the 4th International Particle Accelerator Conference, IPAC-2013, Shanghai, China, 2013 (JACoW, Geneva, Switzerland, 2013), TUPFI011, pp. 1349-1351.

[90] ATLAS Collaboration, Measurement of the total cross section from elastic scattering in pp collisions at $s=$ $\sqrt{8} \mathrm{TeV}$ with the ATLAS detector, Phys. Lett. B 761, 158 (2016).

[91] LHCb Collaboration, Precision luminosity measurements at LHCb, J. Instrum. 9, P12005 (2014).

[92] J. M. Jowett, J.-B. Jeanneret, and K. Schindl, Heavy ion beams in the LHC, in Proceedings of the 2003 Particle Accelerator Conference, Portland, Oregon (IEEE, 
Piscataway, NJ, 2003), TPPB029, pp. 1682-1684, https:// doi.org/10.1109/PAC.2003.1288635.

[93] J. M. Jowett, H. H. Braun, M. I. Gresham, E. Mahner, A. N. Nicholson, E. Shaposhnikova, and I. A. Pshenichnov, Limits to the performance of the LHC with ion beams, in Proceedings of the 9th European Particle Accelerator Conference, Lucerne, 2004 (EPS-AG, Lucerne, 2004), pp. $578-580$.

[94] H. Meier, Z. Halabuka, K. Hencken, D. Trautmann, and G. Baur, Bound-free electron-positron pair production in relativistic heavy-ion collisions, Phys. Rev. A 63, 032713 (2001).

[95] J. M. Jowett, M. Schaumann, and R. Versteegen, Heavy ion operation from Run 2 to HL-LHC, in Proceedings of RLIUP: Review of LHC and Injector Upgrade Plans, Centre de Convention, Archamps, France, 2013 (CERN, Geneva, Switzerland, 2014), pp. 167-184, http://dx.doi .org/10.5170/CERN-2014-006.167.

[96] M. Schaumann, Heavy-ion performance of the LHC and future colliders, Ph.D. thesis, CERN and RWTH Aachen University, 2015.

[97] J. M. Jowett, M. Schaumann, B. Auchmann, C. B. Castro, M. Kalliokoski, A. Lechner, T. Mertens, and C. Xu,
Bound-free pair production in $\mathrm{LHC} \mathrm{Pb}-\mathrm{Pb}$ operation at $6.37 \mathrm{ZTeV}$ per beam, in Proceedings of the 7th International Particle Accelerator Conference, Busan, Korea (JACoW, Geneva, Switzerland, 2016), TUPMW028, pp. 1497-1500, http://dx.doi.org/10.18429/ JACoW-IPAC2016-TUPMW028.

[98] The High Luminosity Large Hadron Collider, edited by O. Brüning and L. Rossi, Advanced Series on Directions in High Energy Physics Vol. 24 (World Scientific, Singapore, 2015).

[99] R. Martin, M. I. Besana, F. Cerutti, A. Langner, R. Tomás, E. Cruz-Alaniz, and B. Dalena, Interaction region design driven by energy deposition, Phys. Rev. Accel. Beams 20, 081005 (2017).

[100] M. I. Besana et al., Energy deposition in the betatron collimation insertion of the $100 \mathrm{TeV}$ Future Circular Collider, in Proceedings of the 8th International Particle Accelerator Conference, Copenhagen, Denmark (JACoW, Geneva, Switzerland, 2017), MOPAB003, pp. 68-71, http://dx.doi.org/10.18429/JACoW-IPAC2017 -MOPAB003. 